\title{
Engineered Capsids for Efficient Gene Delivery to the Retina and Cornea
}

\author{
Amy Frederick, ${ }^{1, \dagger}$ Jennifer Sullivan, ${ }^{1, \dagger}$ Lin Liu, ${ }^{2}$ Matthew Adamowicz, Michael Lukason, Jasmine Raymer, \\ Zhengyu Luo, ${ }^{1}$ Xiaoying Jin, ${ }^{2}$ Kollu Nageswara Rao, and Catherine O'Riordan ${ }^{1, *}$ \\ ${ }^{1}$ Department of Gene Therapy Research, Rare and Neurologic Diseases Therapeutic Area, Sanofi, Framingham, Massachusetts, USA; ${ }^{2}$ Department of BioAnalytics, \\ Sanofi, Framingham, Massachusetts, USA. \\ †These authors contributed equally to this work.
}

\begin{abstract}
Adeno-associated viral (AAV) vectors represent an ideal vehicle for human gene transfer. One advantage to the AAV vector system is the availability of multiple naturally occurring serotypes that provide selective tropisms for various target cells. Strategies to enhance the properties of the natural AAV isolates have been developed and can be divided into two approaches, rational design or directed evolution. The rational design approach utilizes knowledge of AAV capsids to make targeted changes to the capsid to alter transduction efficiency or specificity, while the directed evolution approach does not require a priori knowledge of capsid structure and includes random mutagenesis, capsid shuffling, or random peptide insertion. In this study, we describe the generation of novel variants for both AAV2 and AAV5 using a rational design approach and knowledge of AAV receptor binding, surface charge, and AAV capsid protein posttranslational modifications. The novel AAV2 and AAV5 variants demonstrate improved transduction properties in both the mouse retina and cornea. The translational fidelity of the novel AAV2 variant was confirmed in the context of the nonhuman primate (NHP) retina, whereas a NHP tissue explant model was established to allow the rapid assessment of translational fidelity between species for the AAV5 variants. The capsid-modified AAV2 and AAV5 variants described in this study have novel attributes that will add to the efficacy and specificity of their potential use in gene therapy for a range of human ocular diseases.
\end{abstract}

Keywords: AAV capsid variants, ocular gene therapy, AAV capsid engineering, AAV capsid posttranslational modifications

\section{INTRODUCTION}

THE SUCCESSFUL CLINICAL APPLICATION of adeno-associated viral (AAV) gene therapy vectors for ocular disease has been demonstrated and ocular gene therapy products are now a reality. Notably, the first human gene therapy drug to be approved by the Food and Drug Administration, and to enter the U.S. market, was Luxturna, a treatment for an inherited retinal dystrophy, Leber's congenital amaurosis $2{ }^{1,2}$ Numerous ongoing clinical and nonclinical studies further underscore the utility of the AAV vector system as an effective gene delivery vehicle for the eye, and the availability of a variety of naturally occurring serotypes, displaying various retinal cell tropisms, are considered effective tools to target therapeutic genes to the retina. One disadvantage to the first-generation capsids, in the context of posterior retinal gene therapy, is that their delivery requires an invasive subretinal surgery, thus to address the need for a less-invasive delivery route, research is focused on developing $\mathrm{AAV}$ vectors that can transduce outer retina (i.e., photoreceptors $[\mathrm{PR}] /$ retinal pigment epithelium [RPE]) following intravitreal injection. ${ }^{3,4}$ Moreover, the delivery of genes to the anterior chamber of the eye, specifically corneal endothelial cells, would likewise benefit from a less-invasive surgical procedure than intracameral delivery ${ }^{5}$ or corneal puncture. ${ }^{6}$ Notably, both intravitreal and subretinal delivery of AAV vectors are associated with a dose-dependent inflammation, ${ }^{4,7}$ thus an important advancement to engineering capsids with improved efficiency, is the ability to address immunogenicity and dosage challenges without compromising efficacy.

Strategies to identify next-generation capsids can be divided broadly into two approaches, rational design ${ }^{8}$ and directed evolution, ${ }^{9}$ with the latter approach involving iterative screening of complex AAV capsid libraries to

* Correspondence: Dr. Catherine O'Riordan, Department of Gene Therapy Research, Rare and Neurologic Diseases Therapeutic Area, Sanofi, 49 New York Avenue Framingham 01701, Massachusetts, USA. E-mail: catherine.o'riordan@sanofi.com 
identify capsids with desired attributes. Additionally, the directed evolution approach does not require any knowledge of underlying structure-function relationships in the capsid proteins. In contrast, rational design requires knowledge of the AAV capsid structure and function, and progress using this strategy has been facilitated by the use of X-ray crystallography and cryoelectron microscopy, to solve the structures of first-generation AAVs. ${ }^{10-16}$ Structure knowledge provides a basis for developing customized capsids for specific targeting of AAV vectors. The interaction of domains on the surface of the AAV capsid with specific cellular receptors dictates the tissue tropism for the various AAV serotypes, with different AAV serotypes utilizing unique cellular receptors. For example, the primary receptor for AAV2 is heparan sulfate proteoglycan (HSPG) ${ }^{17}$ whereas the receptors for AAV5 have been identified as $\alpha-2-3-N$-linked sialic acid and platelet-derived growth factor receptor. ${ }^{18,19}$ Previously, we and others, described AAV2 variants devoid of canonical heparan sulfate (HS)-binding residues. These AAV2 variants were shown to mediate very efficient photoreceptor transduction when delivered subretinally, a tropism that is in vast contrast to that observed with the parental, heparan sulfate-binding AAV2, which shows limited tropism for photoreceptor cells with subretinal delivery. ${ }^{20,21}$ Thus, knowledge of structural determinants of receptor binding and tropism, provide insights for modifying the capsid to generate variants with desired transduction profiles.

The AAV capsid is composed of the three structural proteins viral protein (VP)1, VP2, and VP3, which are expressed from the same open reading frame in an approximate stoichiometry of $1: 1: 10$, with an alternative start codon for VP2 and VP3. ${ }^{22-24}$ The capsid proteins share most of their amino acid sequence; VP1 and VP2 differ from VP3 by a shared N-terminal extension of $\sim 65$ amino acids, depending on the serotype, with VP1 containing an additional $\sim 135$ unique amino acids, a region known as VP1-unique (VP1u).$^{10,22-24} \mathrm{VP} 1$ is required for viral infectivity, in part due to the presence of a highly conserved, N-terminal phospholipase A2 (PLA2) homology domain (amino acids 44-138) that is buried within the capsid interior but becomes externalized through pores found at the fivefold symmetry axis following a conformational change in the acidic endosomal compartment. ${ }^{25-}$ ${ }^{27}$ While VP2 is dispensable for capsid assembly and infectivity of the virus, any deletion or mutation in VP1 that results in loss of the phospholipase A2 (PLA2) catalytic domain and its activity, results in a significantly reduced AAV infectivity. ${ }^{25-27}$ In addition, it has been postulated that other signals affecting infectivity might be located on the VP1-unique region of AAV2, which has been reported for several autonomous parvovirus capsid proteins. For example, it has been demonstrated that AAV2 VP1u has protease activity and that the protease site overlaps, in part, with the PLA2 domain in AAV2 VP1u. ${ }^{28}$ Previously, we reported on the development of a direct liquid chromatography/mass spectrometry (LC/MS) intact protein analysis to characterize viral capsid proteins. ${ }^{29}$ Using this method, the complete characterization of the constituent viral capsid proteins of several AAV vectors, including their sequences and posttranslational modifications (PTMs), including acetylation and deamidation were reported. ${ }^{29}$ Notably, the N-termini of all VPs of the six serotypes analyzed (AAV1, 2, 5, 7, 9, and rh10), was confirmed to start at one residue after the predicted $\mathrm{N}$-termini based on DNA sequences, with one exception the VP3 of AAV7. Additionally, the VP1 and VP3 of AAV serotypes $1,2,5,7,9$, and rh10, were shown to contain an $\mathrm{N}$-terminal acetylation. Although $\mathrm{N}$-terminal acetylation of proteins is a widely known phenomenon, the biological significance of $\mathrm{N}$-terminal acetylation of viral capsid proteins is not well understood. One likely hypothesis we proposed, was a potential link between $\mathrm{N}$-terminal acetylation of VP1 and VP3 and viral capsid degradation and uncoating before nuclear entry. ${ }^{29}$

AAV-mediated transduction of multiple retinal cell types in rodent models of inherited retinal disease, after either subretinal or intravitreal injection, is well reported. ${ }^{30,31}$ Additionally, the efficiency and tropism of different AAV serotypes toward photoreceptor cells, have been evaluated in other animal model species, including pigs, ${ }^{32}$ dogs,${ }^{33,34}$ and nonhuman primates (NHPs). ${ }^{35,36}$ One limitation of the direct application of results from animal studies is that viral transduction can vary widely across species. Intravitreal injection in the rodent retina, in contrast to subretinal delivery, ${ }^{37}$ results in robust transduction of retinal ganglion cells, the inner nuclear layer as well as Muller glia (Internal data and Refs. ${ }^{20,38}$ ). However, although some retinal ganglion cells can be transduced in NHPs by intravitreally injected AAV2-based vectors, the area of transduction is restricted primarily to a ring of retinal ganglion cells around the fovea, with no Muller glia cell transduction. ${ }^{39}$ The absence of a cone-enriched fovea in the rat and mouse, further highlights the limitations of using rodent retina to model human retinal disease and the need to confirm translatability of AAV transduction patterns to a larger mammalian retina. Large mammal model availability can be limited, so to assess translatability of AAV transduction patterns, the use of ex vivo mammalian retinal explants is being explored. ${ }^{40}$ The merits of using an organotypic explant approach to test the viral transduction efficiency and tropism of different AAV serotypes in human retina and RPE choroid has been demonstrated, ${ }^{40}$ and while there was evidence of variability in outer nuclear layer (ONL) transduction efficiency across different donor retinas, this approach provides a reliable method to determine how the different AAV capsids transduce a primate retina.

In this study, we describe the use of a rational design approach to engineer AAV capsids with improved trans- 
duction for photoreceptors and corneal endothelial cells. The translational fidelity of our novel heparan sulfate binding knockout (HBKO) variant, AAV2-HBKO, in the NHP retina was confirmed, moreover, the use of an organotypic NHP retinal explant model, to confirm translation of novel AAV5 variants between species, was evaluated. The novel AAV variants described in this study will provide additional tools for gene therapy strategies in the treatment of human blindness.

\section{MATERIALS AND METHODS}

\section{$A A V$ vector production and purification}

AAV vectors were produced using the transient triple transfection method as previously described. ${ }^{41}$ Briefly, HEK293 cells were transfected using polyethyleneimine, and a 1:1:1 ratio of three plasmids (AAV vector, AAV rep/ cap, and Ad helper plasmid). In the case of some AAV production campaigns, the Ad helper functions were replaced by using wtAd5 at a multiplicity of infection (MOI) of 10 . The vector plasmid contains the vector genome (vg) chicken $\beta$-actin hybrid promoter (CBA)-enhanced green fluorescent protein (eGFP) and ITR sequences from AAV2. eGFP expression is driven by the cytomegalovirus enhancer, CBA promoter as described. ${ }^{42}$ The AAV rep/ cap helpers contained rep sequences from AAV2 and serotype-specific capsid sequences with the nomenclature, rep2/cap2, rep2/cap5, rep2/cap7, etc. The pAd helper used was pHelper (Stratagene/Agilent Technologies, Santa Clara, CA). AAV vectors were purified by affinity column chromatography (AVB Sepharose High-Performance medium; GE Healthcare) as previously described. ${ }^{41}$ The fractional content of empty and genome-containing capsids was assessed by analytical ultracentrifugation, as previously described. ${ }^{43}$

\section{Analyzing recombinant AAV vector purity using SYPRO Ruby Protein Gel Stain}

Samples from purified vector were loaded onto a NuPage 4-12\% Bis-Tris gel (Invitrogen). Typically, $1-5 \times 10^{10} \mathrm{vgs}$ of purified vector was analyzed. The gel was stained with SYPRO Ruby Protein Gel Stain (Life Technologies).

\section{LC/MS intact protein analysis}

As previously described, ${ }^{29} \mathrm{AAV}$ virions was first concentrated with an Amicon Ultra centrifugal filter $(0.5 \mathrm{~mL}$, $10 \mathrm{kDa}$ molecular weight cut off [MWCO]) and then washed with $25 \mathrm{mM}$ Tris $\mathrm{pH} 7.1$ three times. The concentrated AAV virions were denatured with $10 \%$ acetic acid vortexed and further diluted with an equal volume of High Performance Liquid Chromatography water. The final acetic acid concentration was 5\%. Fifty microliters of AAV solution ( $\sim 2-5 \mu \mathrm{g}$ of proteins) was injected to Acquity Ultra-Performance Liquid Chromatography (UPLC) coupled with a Xevo G2-XS qTOF MS instrument (Wa- ters, Milford, MA). The separations were performed on a Ethylene Bridged Hybrid (BEH) C8 column (2.1× $100 \mathrm{~mm}$ ) at a flow rate at $250 \mu \mathrm{L} / \mathrm{min}$. Mobile phase A and mobile phase B were $0.1 \%$ formic acid in water and acetonitrile, respectively. The final gradient for $\mathrm{C} 8$ column was as follows: from $10 \% \mathrm{~B}$ to $20 \% \mathrm{~B}$ over $6 \mathrm{~min}$, from $20 \%$ B to $30 \%$ B over $10 \mathrm{~min}$, then from $30 \%$ to $38 \%$ B over $40 \mathrm{~min}$. The capillary voltage and sampling cone voltage of the mass spectrometer were set at $3.5 \mathrm{kV}$ and $45 \mathrm{~V}$, respectively. The mass spectra were acquired in positive sensitivity mode over m/z 500-4,000. MaxEnt1 in MassLynx software version 4.1 was used for protein deconvolution.

\section{Enzymatic digestions of AAV1 and AAV2 VPs}

AAV2-eGFP generated from triple transfection as well as producer cell line (PCL) process ${ }^{41}(\sim 10 \mu \mathrm{g}$ capsid protein) was first concentrated using Amicon Ultra centrifugal filters ( $10 \mathrm{kDa} \mathrm{MWCO})$, denatured with $6 \mathrm{M}$ Guanidine- $\mathrm{HCl}$, and $50 \mathrm{mM}$ Tris at $\mathrm{pH}$ 8.5. The proteins were reduced with $5 \mathrm{mM}$ Dithiothreitol at $60^{\circ} \mathrm{C}$ for $30 \mathrm{~min}$ in darkness and then alkylated with $15 \mathrm{mM}$ iodoacetamide at room temperature for $30 \mathrm{~min}$. The samples were buffer exchanged into $25 \mathrm{mM}$ Tris $\mathrm{pH} 7.1$ for digestion using Bio-Spin ${ }^{\circledR} 30$ Tris column. After buffer exchange, the samples were split into two aliquots. Each aliquot was digested with trypsin at 1:25 or Asp-N at 1:50 enzyme: protein ratio $(w / w)$ for $2 \mathrm{~h}$ at $37^{\circ} \mathrm{C}$, respectively.

\section{UPLC/MS/MS peptide mapping}

The protein digests were analyzed by UPLC/MS/MS ${ }^{\mathrm{E}}$ using an Acquity UPLC-Xevo G2-XS qTOF MS system (Waters). The separation was achieved using a BEH300 C18 column $(2.1 \times 150 \mathrm{~mm})$ with a linear gradient from $2 \%$ to $40 \%$ B ( $0.1 \%$ formic acid in acetonitrile) over $68 \mathrm{~min}$ at a flow rate of $250 \mu \mathrm{L} / \mathrm{min}$. For MS, the capillary voltage and sampling cone voltage were set $3.0 \mathrm{kV}$ and $30 \mathrm{~V}$, respectively. The mass spectra were acquired in the positive sensitivity $\mathrm{MS}^{\mathrm{E}}$ mode in the $\mathrm{m} / \mathrm{z}$ range of $500-2,000$.

\section{Determination of deamidation levels in AAV VPs}

The extracted ion chromatograms of peptides and their corresponding deamidated species were used for calculation of deamidation levels. ${ }^{29}$

\section{Generation of AAV capsid variants}

Deamidation variants N57D and G58D are based on pim45BD-cap2, an AAV helper plasmid that contains both rep and cap sequences from AAV2. Fragments containing the designated mutations were synthesized (Genscript) and subcloned into pim45BD-cap2. Mutations were verified by DNA sequencing (Genewiz). Acetylation variants were constructed as above, using pHLP19-cap5.2, a helper plasmid that contains rep from AAV2 and cap from 
AAV5, as the parental plasmid. AAV2-HBKO and AAV5 arginine mutant capsid plasmids were generated by sitedirected mutagenesis using the QuikChange Lightning Site-Directed Mutagenesis Kit (Agilent Technologies), according to the manufacturer's protocol. The pIM45BD plasmid was used to generate AAV2-HBKO using a polymerase chain reaction (PCR) mutagenesis primer designed to alter the codons encoding arginine 585 and 588 on VP3 to alanine. The sequence of the mutagenic primer used to generate the R585A and R588A mutations was: TATCTACCAACCTCCAGGCAGGCAACGCAC AAGCAGCTACCGCAG. The AAV5-G474R, AAV5N564R, and AAV5-N573R mutants were generated using the AAV5 rep/cap plasmid (pHLPcap5.2) as a template and PCR mutagenic primers designed to change the respective arginines to alanines. The sequence of the mutagenesis primer used to introduce the G474R mutation was CCAGGTTCCAGCGCTGGGTTCGGCC. The sequence of the mutagenesis primer used to introduce the N564R mutation was CCGCGTGGCGTACCGCGTC GGCGGGCAG. The sequence of the mutagenesis primer used to introduce the N573R mutation was CAGTGGT GGAGCTCTGTCTGTTGGTGGCCATCTG. All mutations were confirmed by DNA sequencing.

\section{Infectious AAV particle titration}

Titration of infectious AAV particles by quantitative PCR (qPCR) was performed using the endpoint dilution assay as described previously. ${ }^{44}$ Tissue culture infective dose (TCID)50 titers were calculated from two independent assays and results are shown in Supplementary Table S1. Briefly, HeLa RC 32 cells (ATCC ${ }^{\circledR}$ CRL-2972 ${ }^{\mathrm{TM}}$ ) harboring integrated copies of both rep and cap genes, were infected with 10-fold dilutions of the AAV vector preparations, in the presence of wild-type Ad5 (MOI 10). Infectious titer was calculated as IU/mL using qPCR assay targeting the bovine growth hormone poly A sequence encoded in the vg. Additionally, titration of infectious AAV particles was also assessed by transgene expression. ${ }^{44}$ HeLa cells were infected with serial dilutions of AAV vectors. Analysis of GFP expression by fluorescence microscopy of cells was performed with the following limit of quantitation (LOQ) and limit of detection (LOD) and results are shown in Supplementary Table S1.

LOQ: Detectable signal in wells but not quantifiable (too few wells positive).

LOD: No detectable signal in any wells $(<1,000 \mathrm{IU} / \mathrm{mL})$.

\section{NHP studies}

The NHP study was performed at Covance Laboratories, Inc. (Madison, WI). Four adult male cynomolgus monkeys (Macaca fascicularis) were screened for neutralizing antibodies against AAV5 and AAV2 before surgery as previously described. ${ }^{35}$ Animals considered seronegative (serum titers $\leq 1: 4$ ) were included in the study. Cynomolgus monkeys were assigned to two groups and were administered either AAV2-HBKO-eGFP or AAV5-eGFP. Animals were dosed through subretinal injection to both eyes (OU) once on day 1 of the dosing phase at a volume of $120 \mu \mathrm{L} /$ eye. The diluent was Alcon ${ }^{\circledR}$ BSS $^{\circledR}$ with $0.014 \%$ polysorbate 20 . Dosing syringes (1.0 mL Luer Lok ${ }^{\mathrm{TM}}$ Becton Dickinson Product 309628 or equivalent) were filled and affixed to a Dutch Ophthalmic Research Center (DORC) 23-gauge needle with an extendible 41-gauge subretinal injection needle on the day of dosing using aseptic procedures under a laminar flow hood. Injections were administered within $30 \mathrm{~min}$ of filling the DORC injector. Dosing was done performed in OU of each animal. Animals were anesthetized with intramuscular injections of atropine $(0.01 \mathrm{mg} / \mathrm{kg})$, ketamine $(2-10 \mathrm{mg} / \mathrm{kg})$, and dexmedetomidine $(0.25 \mathrm{mg} / \mathrm{kg})$. Following the procedure, the anesthesia was reversed with atipamezole $(0.25 \mathrm{mg} / \mathrm{kg})$. The anesthesia regimen was adjusted based on the responsiveness of animals and according to Covance veterinary staff. Eyes were cleaned with an $\sim 1 \%$ povidone iodine solution (prepared with sterile saline and $5 \%$ povidone iodine) and rinsed with sterile saline. An $\sim 2.5 \%$ povidone iodine solution was used at the dose site before injection. The injection was performed following a study-specific procedure, briefly described as follows.

Pupils were dilated with a topical mydriatic agent. The DORC disposable dual-bore injection needle (23-gauge) was introduced directly through the sclera in the superior temporal quadrant of the globe $\sim 3 \mathrm{~mm}$ posterior to the corneal limbus and moved through the vitreous under visual control using a surgical microscope viewing through a dilated pupil, with a modified fundus-viewing lens placed on the cornea. The 41-gauge cannula tip was advanced from the 23-gauge needle and gently touched the retinal surface. The dose was injected through the neural retina into the subretinal space, resulting in a subretinal bleb. The 41-gauge cannula tip was retracted, and the 23-gauge needle was withdrawn. A topical antibiotic and steroid ointment (Neo-poly-dex) was instilled in each eye following all other postdose ocular procedures. The injection occurred in a small portion of the retina in the mid-arcade region.

\section{Fundus autofluorescence imaging}

The fundus autofluorescence (FAF) imaging was done once during the predose phase and during weeks 2, 4, and 6 of the dosing phase. Animals were anesthetized (remained under anesthesia used for dosing) for FAF imaging. Eyes were dilated with a mydriatic agent (1\% tropicamide). FAF images were taken of each eye to include the subretinal dose sites and the fovea. Images were taken with a Heidelberg SPECTRALIS ${ }^{\circledR}$ instrument.

\section{Tissue processing of NHP retina}

On day 43 of the dosing phase, all animals, having been fasted overnight, were anesthetized with sodium pento- 
barbital, exsanguinated, and necropsied. The eyes from each animal were injected with and submerged in chilled $4 \%$ paraformaldehyde (PFA) and stored in a refrigerator, set to maintain $2-8^{\circ} \mathrm{C}$, for $48-72 \mathrm{~h}$. The eyes were then embedded in paraffin, sectioned, and slides were prepared for immunohistochemistry (IHC) analysis. From the temporal calotte, 20 serial sections were taken from the fovea. In addition, 20 serial sections were taken every $250 \mu \mathrm{m}$ for a total of eight steps from the remaining temporal calotte. One slide from the fovea and one slide from each of the eight steps of the temporal calotte were stained with Hematoxylin and Eosin.

\section{Immunohistochemistry analysis}

A single slide through the fovea of each eye was examined by bright field microscopy. The presence or absence of eGFP expressing photoreceptors was noted at the time of observation. The antibody that identified eGFP was tagged with a chromogen that produced a brown precipitate. The antibodies that identified rhodopsin were tagged with a chromogen that produced a red precipitate. The presence or absence of rod photoreceptors that expressed eGFP was noted. Selected representative images were recorded. To assess the eGFP expression, we performed immunohistochemistry. Briefly, sections were washed with xylene three times for 5 min each. Sections were rehydrated by washing in graded series of alcohol, rehydrated in distilled water. Following antigen retrieval, endogenous peroxidase activity was blocked with hydrogen peroxide and nonspecific protein binding with normal goat serum. Sections were incubated with eGFP and rhodopsin antibodies. After washing, sections incubated with ChromoPlex 1 Dual Detection for bond (Leica, Wetzlar, Germany) were used for the visualization of dual histochemical staining, according to the manufacturer's instructions. Sections were counterstained with Hematoxylin, which were then dehydrated with ethanol before mounting. Paraffin-embedded sections were deparaffinized and rehydrated in a graded ethanol. Antigen retrieval was performed, and sections were blocked with protein block serum-free reagent (Dako). Sections were then incubated with mouse anti-eGFP and were incubated overnight at $4^{\circ} \mathrm{C}$ and then washed, and incubated with anti-mouse Alexa Fluor 488 secondary antibody. The sections were washed in phosphate-buffered saline (PBS), and coverslipped using mounting solution and imaged using microscope.

\section{Immunofluorescence}

The remaining slide was labeled for eGFP and 4',6diamidino-2-phenylindole (DAPI) (immunofluorescence). The slides were stained for the immunofluorescence detection of eGFP and costained with DAPI to visualize the nuclei.

\section{Photoreceptor cell counting and quantification of transduction}

The step-sectioned slide set from each eye was reviewed and the slide that contained the fovea was selected for analysis. The photoreceptor layer was imaged from one border of the subretinal bleb to the other. Morphometric analysis was performed using NIH ImageJ (version 1.49t) to determine the percentage of photoreceptors expressing the eGFP transgene within the borders of the subretinal bleb. Color balance command was used to adjust the brightness and eliminated background fluorescence. The surface area of ONL was measured with measuring command. Then we used thresholding to create a mask of GFP-expressing cell bodies and measured surface area. The percentage of transduced photoreceptors was determined using the formula: Percentage of Transduced Photoreceptors $=P R$ transduced $/ P R$ total $\times 100$. The result from each microscope image was plotted. The total percentage was calculated by taking the sum of the areas of the PR transduced and PR total using the above formula.

\section{Mouse studies}

All animal studies were performed according to a detailed protocol, approved by Sanofi's Internal Animal Care Use Committee. At postnatal day P45, mice were placed under general anesthesia with intraperitoneal injection of ketamine $(90 \mathrm{mg} / \mathrm{kg}) / x y l a z i n e(9 \mathrm{mg} / \mathrm{kg})$. Pupils were dilated with topical application of $1 \%$ tropicamide (Akorn Pharmaceuticals, Lake Forest, IL). To perform subretinal injections, aliquots of AAV were thawed on ice, fluorescein (AK-FLUOR, 10\%-Akorn Pharmaceuticals) was added to the viral preparation to aid in visibility of AAV delivery. Under visualization with an operating surgical microscope (PSMT5N; World Precision Instruments, Sarasota, FL), an incision was made through the sclera immediately posterior to the nasal limbus using a beveled 30 -gauge needle. Through this incision, a 35-gauge blunt needle (NF35BL; World Precision Instruments), which is mounted in to the SilFlex tubing connected to NanoFil syringe, was introduced into the subretinal space. Viral suspension was injected over $20 \mathrm{~s}$ into the subretinal space using a programmable microsyringe pump with foot pedal control (UMP3, UltraMicroPump; World Precision Instruments). The needle was held in place for at least $20 \mathrm{~s}$ after completion of the injection. Fundus and OCT examination was performed following injection to visualize the location of injection and thus ensure that AAV vectors were injected into the subretinal space. A 3.5\% Akten (lidocaine hydrochloride ophthalmic gel; Akorn Pharmaceuticals) Ophthalmic gel was applied to the cornea as a topical anesthetic. A small amount of neomycin/polymyxin B/dexamethasone ophthalmic ointment (Alcon Laboratories, Inc., Fort Worth, TX) was spread over the eye before placing the animal in a $37^{\circ} \mathrm{C}$ incubator to recover from anesthesia. For intravitreal injections, mice were anesthetized, and eyes were dilated same as described above. One microliter of AAV suspension was injected into the vitreous through sclera, which is $1 \mathrm{~mm}$ from the limbus using $35 \mathrm{G}$ beveled-tip needle attached to a 
10- $\mu \mathrm{L}$ Nanofil syringe through SilFlex tubing (World Precision Instruments). OCT was performed immediately to check for any retinal damage following injections. A small amount of Akten and triple antibiotic ophthalmic ointment was applied on the eye before placing at $37^{\circ} \mathrm{C}$.

\section{Quantification of recombinant AAV transduction in mouse retinal lysates}

Following euthanasia, eyes were collected, frozen immediately on dry ice, and stored at $-80^{\circ} \mathrm{C}$ for future dissection. All dissection steps were performed under a dissecting microscope using cold instruments, while maintaining the eye in a frozen state during dissection. The anterior segment was removed using a razor, excess tissue was removed from the back of the eye if needed, and the lens were then removed. Retinal lysates were generated by placing the frozen vitreous humor, retina, and eye cup in $200 \mu \mathrm{L}$ of cell lysis buffer from the GFP EnzymeLinked Immunosorbent Assay (ELISA) Kit and homogenized at $4^{\circ} \mathrm{C}$ (Fisher Bead Mill). The eGFP protein was quantified using the GFP ELISA Kit (cat. no. ab171581; Abcam). Total protein levels were quantified using the BCA Protein Assay Kit (Pierce). The levels of eGFP were normalized to total protein. Genome titers were determined by quantitative real time PCR (7500 Real-Time PCR System; Applied Biosystems) using TaqMan Universal Master Mix (Thermo Fisher) with primers specific for the polyadenylation signal. Vector levels were expressed as genomes per microgram protein.

\section{Micron IV in-life imaging}

At 4 weeks postinjection, animals were anesthetized using $120 \mathrm{mg} / \mathrm{kg}$ ketamine and $6.7 \mathrm{mg} / \mathrm{kg}$ xylazine delivered intraperitoneally. OU were dilated using $\sim 1-3$ drops of $1 \%$ Mydriacyl (Alcon) administered topically $\sim 10 \mathrm{~min}$ before testing to allow for complete mydriasis. A small drop of goniotaire was placed on the cornea to avoid dehydration. Mice were placed on a platform and their eyes were aligned to a Micron IV fundus camera. Images were taken once the optic nerve head was visible and were focused on the retina. For capturing GFP fluorescence, we used the same gain settings across all samples. Anesthetics were reversed by injecting atipamezole ("Antisedan") and mice were placed on warm pad until they recovered.

\section{NHP retinal explants}

All animal procedures were conducted in compliance with the Animal Welfare Act, the Guide for the Care and Use of Laboratory Animals, the Office of Laboratory Animal Welfare, and in accordance with the Association for Research in Vision and Ophthalmology Statement for the Use of Animals in Ophthalmic and Vision Research. Fresh monkey eyes without known ocular diseases were obtained from Biomere (Worcester, MA), where they were enucleated $15 \mathrm{~min}$ after animals were sacrificed. The eyes were placed in Neurobasal medium and transported immediately on ice. Under aseptic conditions, all extraocular connective tissues were removed, and the eyes were disinfected with $70 \%$ ethanol followed by washing with PBS. Before starting the experiment, six-well transwell culture plates were preincubated with $2 \mathrm{~mL}$ of complete Neurobasal medium (cat. no. 21103049; Thermo Fisher Scientific, Waltham, MA), supplemented with $1 \% \mathrm{~N}-2$ Supplement (cat. no. 17502048; Thermo Fisher Scientific), 2\% B-27 Supplement (cat. no. 17504044; Thermo Fisher Scientific), 1\% GlutaMAX Supplement (cat. no. 35050-061; Thermo Fisher Scientific), $0.2 \mu \mathrm{g} / \mathrm{mL}$ of recombinant human $\beta$-nerve growth factor (cat. no. 256-GF100; R\&D Systems, Minneapolis, MN), and $0.4 \mu \mathrm{g} / \mathrm{mL}$ recombinant human epidermal growth factor (cat. no. 236EG-200; R\&D Systems) beneath the transwell insert and $0.5 \mathrm{~mL}$ in the transwell insert in humidified cell culture incubators at $37^{\circ} \mathrm{C}$ and $5 \%$ carbon dioxide $\left(\mathrm{CO}_{2}\right)$. An incision was made with an $18 \mathrm{G}$ needle around $5 \mathrm{~mm}$ below the limbus. By using this incision as an entry point for the scissors, the anterior part of the eye, cornea, lens, and vitreous, were removed from each eye leaving posterior eyecups consisting of intact neural retina, choroid, and sclera. Next, three flaps were made to open the eye cup by making three cuts toward the optic nerve head. While the whole eye cup is submerged in the Neurobasal complete medium, an $8 \mathrm{~mm}$ biopsy punch was used to cut equatorial, full-thickness posterior segment explants. The retina was subsequently peeled off by gently applying a piece of dry sterile filter paper onto the ganglion cell layer, lifting off the neural retina, and placing the filter paper with attached retina onto the culture insert, photoreceptors facing down, and filter paper was gently removed with fine forceps. After $24 \mathrm{~h}$, the medium was replaced with fresh complete Neurobasal medium and half of the AAV was injected directly beneath each retinal explant, creating a bleb similar to what is formed in vivo when performing therapeutic subretinal injections. An additional half of virus was added to the culture medium that was placed beneath the transwell insert.

Finally, the explants are incubated at $37^{\circ} \mathrm{C}$ and $5 \% \mathrm{CO}_{2}$. AAV vectors at a dose of $1.8 \times 10^{11} \mathrm{vgs}$ was used for each explant. The medium was changed every other day and cultures were maintained for 6 days posttransduction. After 7 days of culture, retinal explants were rinsed in $1 \times \mathrm{PBS}$ and fixed for $3 \mathrm{~h}$ in $4 \%$ PFA. Explants were washed three times with PBS to remove the residual PFA and cryoprotected in graded sucrose 10-30\%, after which they were frozen in optimum cutting temperature compound at $-80^{\circ} \mathrm{C}$. Thirteen micrometer-thick sections were cut using a cryostat (CryoStar NX70 cryostat; Thermo Fisher Scientific) and mounted with VECTASHIELD DAPI (Vector Laboratories, Peterborough, United Kingdom). The native eGFP expression was observed and images were captured using an inverted fluorescence microscope (Axio Observer 
Z1; Carl Zeiss, Inc., Oberkochen, Germany) using appropriate excitation and detection settings.

\section{Statistical analyses}

All data shown in the present article are reported as mean \pm standard deviation. GraphPad Prism 7.0 software was used for statistical analyses. The statistical tests used were unpaired Student's $t$-test for two-group comparisons. $p<0.05$ was considered significant. The statistical analyses performed for each dataset is indicated in the figure legends. For all figures, ${ }^{*} p<0.05,{ }^{* *} p<0.01,{ }^{* *} p=0.001$, $* * * * p<0.0001$.

\section{RESULTS}

\section{Translational fidelity of AAV2-HBKO in the NHP retina}

Previously, we reported on the transduction activity of a novel AAV2 capsid variant in the mouse retina. ${ }^{20}$ The AAV2 variant had amino acids R585A and R588A mutated, which are required for AAV2 binding to its receptor, HSPG, to generate a variant known as AAV2-HBKO. In contrast to parental AAV2, the AAV2-HBKO variant displayed low transduction activity following intravitreal delivery to the mouse eye; however, following its subretinal delivery, AAV2-HBKO resulted in significantly greater photoreceptor transduction. Frequently, the AAV transduction profile in mouse is not always predictive of transduction potential in the NHP, thus, the performance of AAV2-HBKO in the NHP retina was evaluated to determine if this novel variant demonstrated a similar improvement in retinal transduction in a primate. The objective of the study was to compare eGFP expression in photoreceptors from an AAV5 and an AAV2-HBKO vector, when administered as a single dose through subretinal injection to male cynomolgus monkeys. Male cynomolgus monkeys ( $M$. fascicularis) were assigned to two groups, and AAV5-eGFP or AAV2-HBKO-eGFP were administered at a dose of $1 \times 10^{12} \mathrm{vg} /$ eye as described in Table 1 . The vector preparations were analyzed using a series of optimized assays to confirm quality (Supplementary Fig. S1).

Six weeks postvector administration, the animals were euthanized and the eyes were processed for histological sectioning. The section that passed through the fovea was selected for analysis (Fig. 1A). The assessment of eGFP expression in photoreceptors, following administration of AAV5-eGFP or AAV2-HBKO-eGFP, was evaluated by
FAF and IHC. Both vectors harbored the same expression cassette, which consisted of a human rhodopsin promoterdriving expression of eGFP. The animals were euthanized $\sim 6$ weeks following vector administration and the eyes processed for paraffin embedding and histological sectioning. The slides were stained for the immunofluorescence detection of eGFP (Fig. 1B), and images were sequentially collected from one edge of the subretinal bleb to the other. Expression of the eGFP transgene appeared to be uniform across the subretinal bleb. In areas where there were fewer rod photoreceptors (adjacent to and within the fovea), the transgene expression was diminished, confirming the fidelity of the human rhodopsin promoter in restricting expression to the rod photoreceptors. Previously, AAV5 was shown to transduce cone photoreceptors when transgene expression was under the control of the rod and cone-specific human rhodopsin kinase promoter. $^{45}$ Quantification of the percentage of photoreceptor transduction under the bleb revealed that AAV5 and AAV2-HBKO demonstrate equal photoreceptor transduction activity in the NHP retina (an average of $61 \%$ of the photoreceptors were transduced by both AAV5 and AAV2-HBKO) (Fig. 1C).

Additionally, the expression of eGFP was monitored using spectral domain optical coherence tomography with autofluorescence imaging capabilities; eGFP was observed 2 and 4 weeks following vector administration. The intensity of eGFP signal increased with time and was confined to the retina, within the margin of the subretinal bleb in the eyes treated with AAV5-eGFP (Fig. 2A), while in contrast, expression of eGFP from the eyes treated with AAV2-HBKO-eGFP extended well past the margin of the subretinal bleb (Fig. 2B). Separate slides were costained with an anti-eGFP antibody and anti-rhodopsin antibody for the immunohistochemical detection of eGFP as well as rhodopsin; this analysis was done to confirm that only rod photoreceptors were transduced. Figure 3 shows that histological survey of the subretinal bleb. Immunohistochemistry for rhodopsin (red) and eGFP (brown), in paraffin-embedded tissue, reveals that the transduction with the AAV5 vector does not spread from the margin of the subretinal bleb, the transition at the margin is abrupt (Fig. 3A). In contrast, AAV2HBKO-eGFP transduction spreads beyond the bleb and tapers off in areas not lifted by the injection process (Fig. 3B; Supplementary Fig. S2). Colocalization of eGFP and rhodopsin indicate that the transduced cells are rod

Table 1. Description of dosing regimen for subretinal delivery of either AAV5-eGFP or AAV2-HBKO-eGFP to nonhuman primates

\begin{tabular}{cccccc}
\hline Group & No. of Males & Eye & Treatment & Dose Level (vg/Eye) & Dose Concentration $(\mathrm{vg} / \mathrm{mL})^{p}$ \\
\hline 1 & 2 & OU & AAV5-eGFP & $1.2 \times 10^{11}$ & $1.0 \times 10^{12}$ \\
2 & 2 & OU & AAV2-HBKO-eGFP & $1.2 \times 10^{11}$ & $1.0 \times 10^{12}$ \\
\hline
\end{tabular}

${ }^{a}$ Both eyes of each animal were dosed. Animals were dosed at a volume of $120 \mathrm{uL} / \mathrm{eye}$.

${ }^{b}$ Dose concentrations were based on the test article as supplied.

AAV, adeno-associated viral; eGFP, enhanced green fluorescent protein; HBKO, heparan sulfate binding knockout; OU, both eyes; vg, vector genome. 

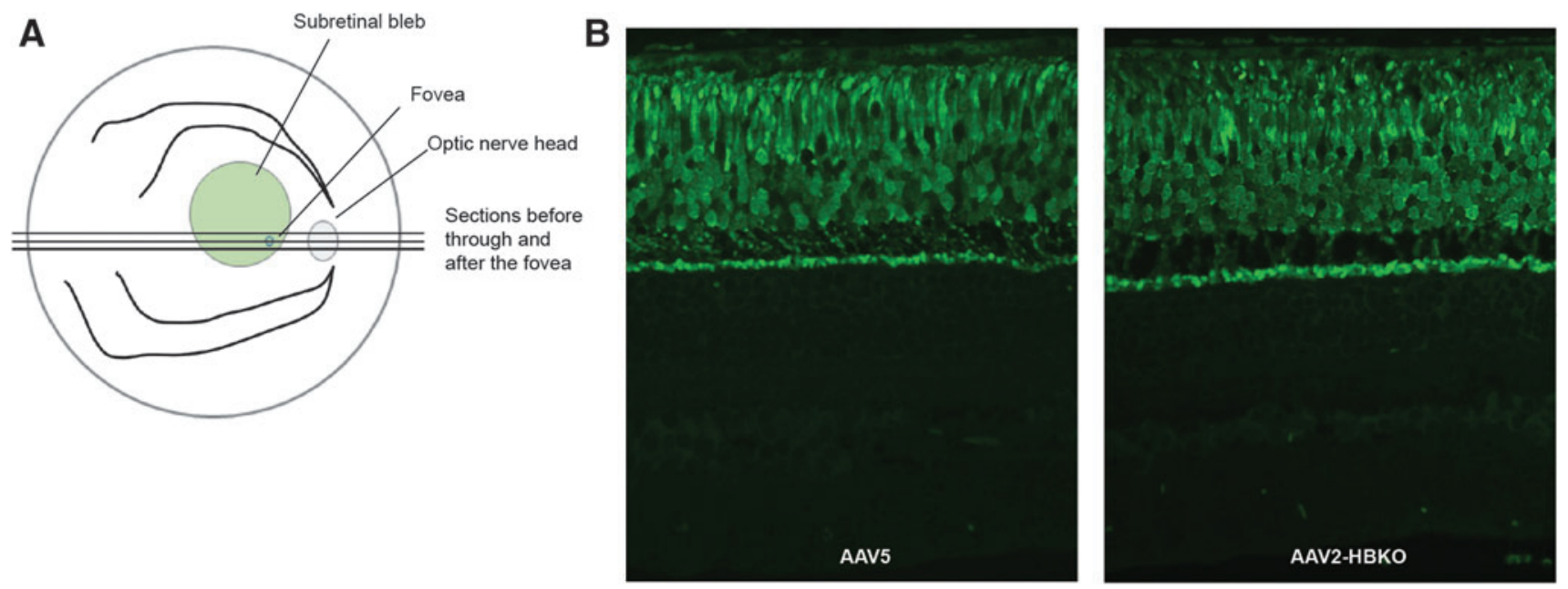

\begin{tabular}{|c|c|c|c|c|}
\hline Group & $\begin{array}{c}\text { Treatment } \\
\text { (vg/eye) }\end{array}$ & Animal ID & $\begin{array}{c}\text { eGFP positive PR } \\
\text { (\% transduced PR } \\
\text { under bleb) OD } \\
\end{array}$ & $\begin{array}{l}\text { eGFP positive PR } \\
\text { (\% transduced PR } \\
\text { under bleb) OS }\end{array}$ \\
\hline \multirow[b]{2}{*}{1} & \multirow{2}{*}{$\begin{array}{c}\text { AAV5 } \\
1.2 \times 10^{11}\end{array}$} & P0001 & 41.7 & 50.1 \\
\hline & & P0002 & 77.1 & 76.1 \\
\hline \multirow[b]{2}{*}{2} & \multirow{2}{*}{$\begin{array}{c}\text { AAV2- HBKO } \\
1.2 \times 10^{11}\end{array}$} & P0101 & 59.8 & 59.1 \\
\hline & & P0102 & 50.9 & 75.9 \\
\hline
\end{tabular}

Figure 1. AAV2-HBKO- and AAV5-mediated eGFP expression in the NHP retina 6 weeks postsubretinal injection. (A) Schematic representation of sections collected for eGFP expression. The black lines represent the areas on the sections that were analyzed for native eGFP expression (before, through, and after fovea). Only the section that passed through the fovea and the bleb was analyzed. (B) Image of photoreceptor cells transduced with AAV5-eGFP or AAV2HBKO-eGFP. (C) Percentage of transduced photoreceptors within the area of the subretinal bleb. AAV, adeno-associated viral; eGFP, enhanced green fluorescent protein; HBKO, heparan sulfate binding knockout; NHP, nonhuman primate; OD, right eye; OS, left eye; PR, photoreceptor; vg, vector genome.

A
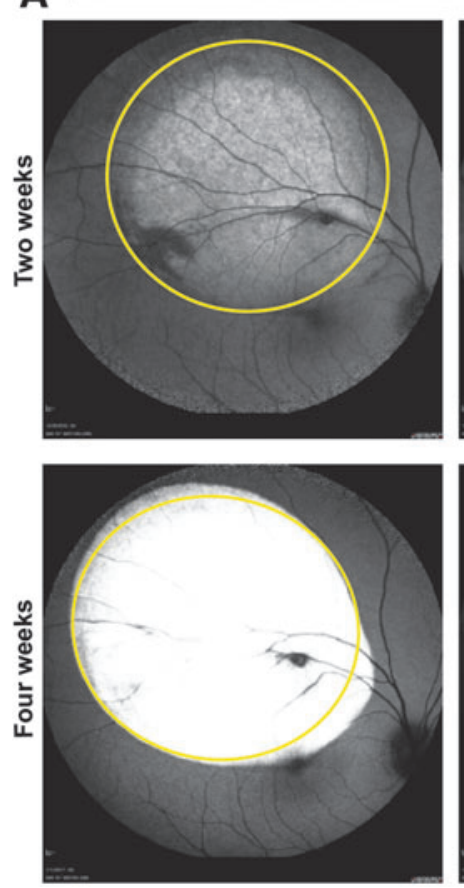

AAV5
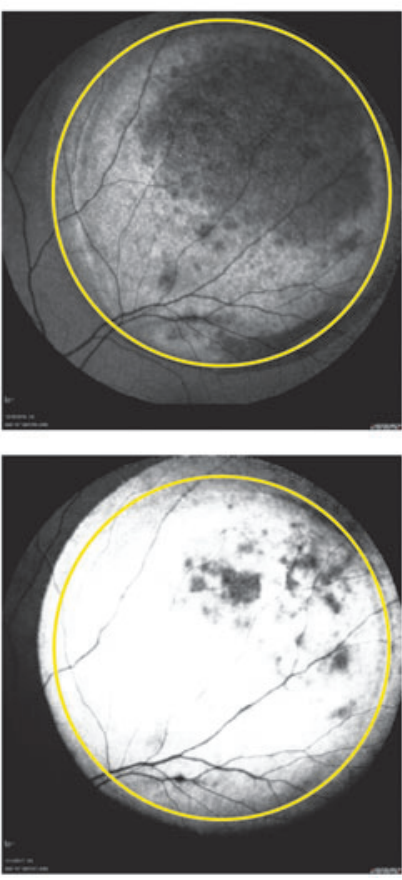

B
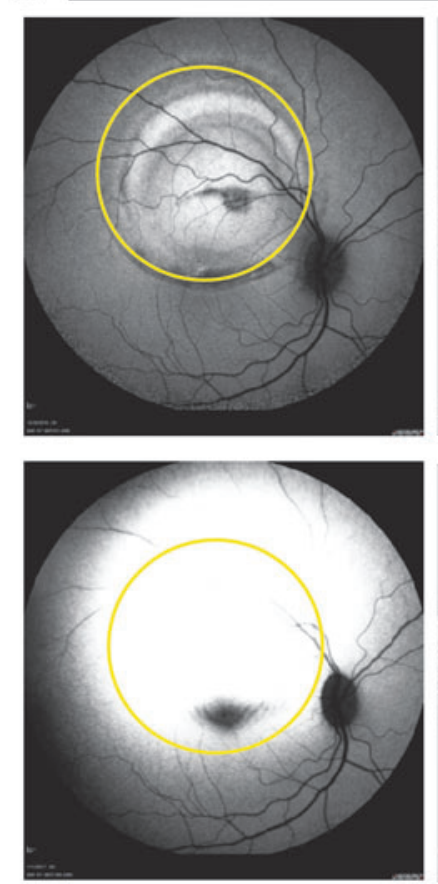

AAV2- HBKO
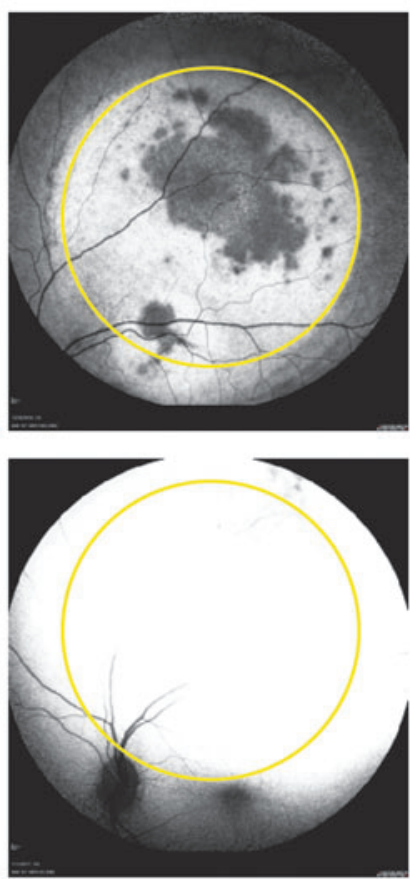

Figure 2. AAV-mediated eGFP expression in NHP eyes by FAF. FAF showing the eGFP fluorescence in the bleb area (yellow circle) treated with AAV5-eGFP (A) or AAV2-HBKO-eGFP (B). An evident increase in eGFP expression was observed at 4 weeks following AAV treatment (A, B). AAV2-HBKO-eGFP vector spread beyond the margins of the subretinal bleb, whereas the AAV5-eGFP vector stayed within the margins of the subretinal bleb (A, B). FAF, fundus autofluorescence. 

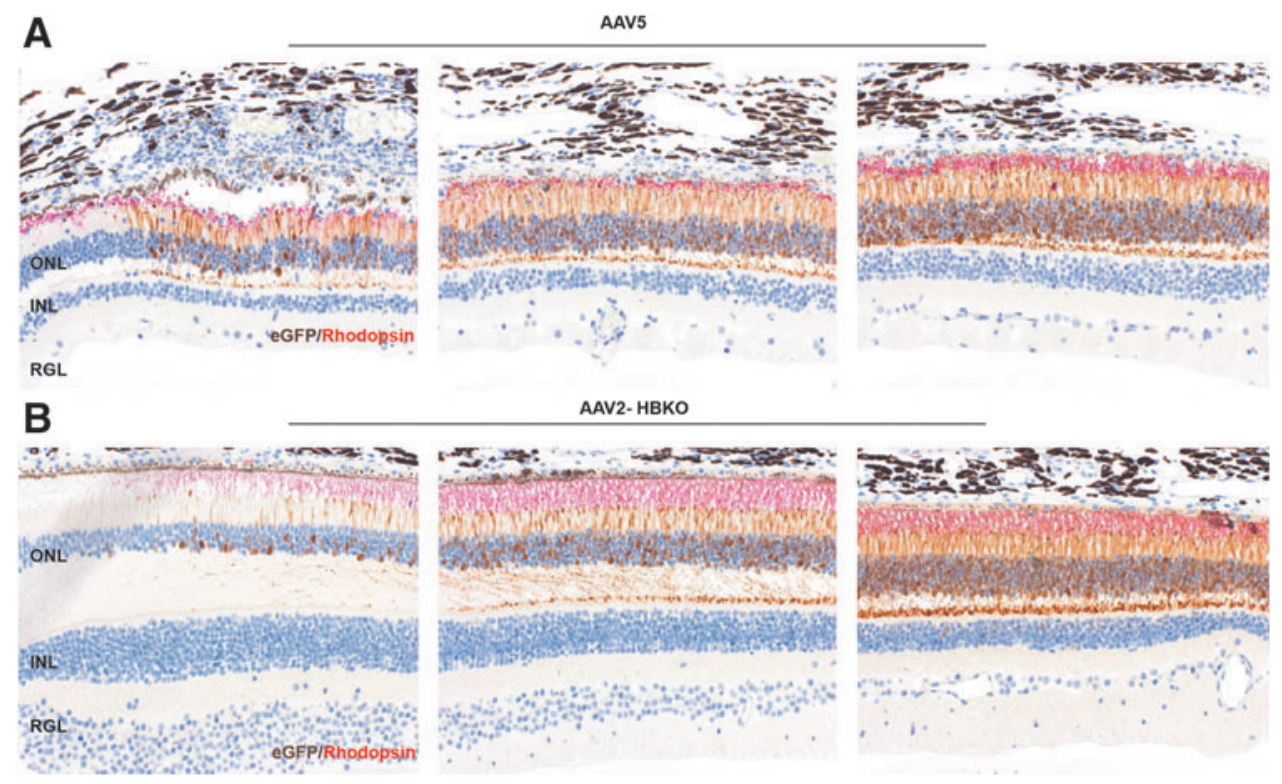

Figure 3. Subretinal injections in NHP show superior transduction ability of AAV2-HBKO over the AAV5 in retina. Relative transduction efficiencies of AAV5 and AAV2-HBKO in rod photoreceptors were compared following subretinal injection. Paraffin-embedded retinal sections were immunolabeled with anti-GFP antibody and anti-rhodopsin antibody for the detection of eGFP (brown) as well as rhodopsin (red). Immunohistochemistry analysis around the bleb region showed that the transduction of the AAV5 vector does not appear to spread from the margin of the subretinal bleb, the transition at the margin is abrupt (A). In contrast, AAV2-HBKO-eGFP was highly efficient at transducing photoreceptors and displayed ability to spread from the bleb and tapers off in areas not lifted by the injection process (B). The AAV transduction was restricted to rod photoreceptors. INL, inner nuclear layer; ONL, outer nuclear layer; RGL, retinal ganglion cell layer.

photoreceptors. The overall architecture of the retina is preserved, although there are changes to the RPE, including hypertrophy, displacement of pigment, and displacement of cells. These signals of intolerability are likely due to expression of the foreign eGFP reporter protein.

\section{Evaluation of the transduction potential of AAV5 arginine variants in the retina}

The AAV2-HBKO variant revealed the importance of arginines, and by extension surface charge, on transduction activity in the retina. The effect of altering surface arginines was further explored with another capsid, AAV5, a serotype that has a high affinity for photoreceptors when delivered subretinally. ${ }^{43}$ AAV5 variants AAV5G474R, AAV5N564R, and AAV5N573R were generated and their tropism in the mouse retina, following intravitreal and subretinal delivery, was evaluated. The surface map of AAV2 was aligned to that of AAV5, ${ }^{14}$ and amino acids in the AAV5 capsid were selected for mutation, to generate arginine-rich AAV5 variants. Of note, the AAV5 variants produced at yields that were comparable or twofold less than yields achieved with parental AAV5, but variants retained the same capsid protein ratio as parental AAV5 (Supplementary Fig. S3). First, subretinal delivery of the AAV5 arginine variants was evaluated in the wild-type mouse retina and their transduction activity compared with that of the parental AAV5 capsid. Figure 4A and $\mathrm{C}$ shows the performance of AAV5, AAV5G474R, AAV5N564R, and AAV5N573R follow- ing subretinal delivery of $1 \times 10^{9}$ vgs of each vector harboring the identical CBA-eGFP expression cassette. Notably, there was no apparent difference in the ability of the AAV5 arginine variants to transduce the retina following subretinal delivery, when compared with parental AAV5 as evidenced by in-life fundoscopic (Fig. 4A) and fluorescence microscopic analysis (Fig. 4C). Analysis of native eGFP fluorescence in transduced retina revealed that the AAV5G474R, AAV5N564R, and AAV5N573R variants transduced the ONL and RPE cells, to the same level as parental AAV5-eGFP (Fig. 4C). Additionally, the AAV5 arginine variants were further evaluated following intravitreal delivery to the mouse retina, at the same vector dose of $1 \times 10^{9}$ vgs/eye. Following intravitreal delivery, the AAV5 variants demonstrated minimal transduction of the retina compared with AAV2 revealed by Micron IV in-life fundoscopic analysis (Fig. 4B). The eGFP fluorescence analysis of the transduced retinae (Fig. 4D), confirmed that all AAV5 arginine variants, AAV5G474R, AAV5N564R, and AAV5N573R, had acquired a novel tropism for corneal endothelial cells, and in contrast, parental AAV5 showed no transduction activity with intravitreal delivery (Fig. 4D).

\section{Probing the role of acetylation on AAV transduction}

The AAV2-HBKO and AAV5 arginine variants were generated using a rational design approach, using knowledge of receptor binding and surface charge, respectively. 

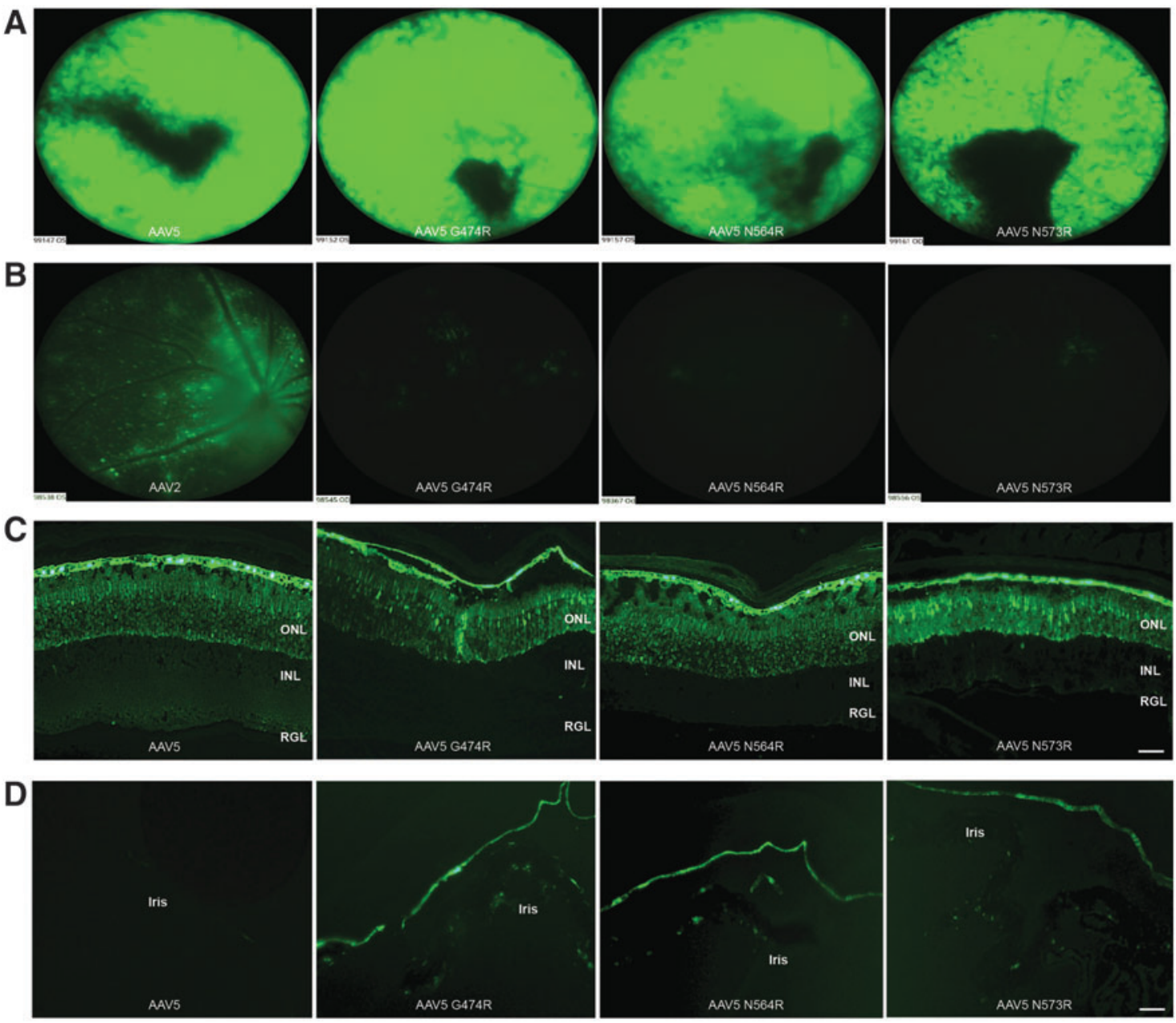

Figure 4. Comparison of retinal and corneal transduction efficiency between AAV5 and AAV5 arginine variants. (A) Micron IV in-life fundus images of WT mice injected subretinally with $1 \times 10^{9}$ vgs of AAV5, AAV5G474R, AAV5N564R, and AAV5N573R eGFP-expressing vectors. Images were taken 4 weeks postinjection of vector. (B) Micron IV in-life fundus images of WT mice were injected intravitreally with $1 \times 10^{9}$ vgs of AAV2, AAV5G474R, AAV5N564R, and AAV5N573R eGFP-expressing vectors. Images were taken 4 weeks postinjection of vector. (C) Cryosections were analyzed for AAV-mediated native eGFP expression under fluorescence microscope. Subretinal injections showed similar transduction efficiency between AAV5 and AAV5 arginine variants 4 weeks postinjection. (D) Transduction efficiency of AAV5 and AAV5 arginine variants in the mouse cornea. AAVs were delivered by intravitreal injection and tissues were analyzed 4 weeks postinjection. Histological sections showed the intense eGFP expression in corneal endothelial cells following intravitreal delivery of the AAV5-eGFP arginine variants. Limited detectable eGFP expression was observed following intravitreal delivery of AAV5. Scale bar $50 \mu \mathrm{m}$.

Next, additional variants were generated using knowledge we gained from LC/MS analysis of the AAV capsid, which identified novel PTMs, including N-terminal acetylation on VP1 and VP3 capsid proteins. ${ }^{29}$ To further explore this attribute, a series of AAV5 acetylation variants was generated to elucidate the role of this significant PTM on AAV5 biology in the retina. The mutations that were introduced into the AAV5 capsid sequence are described in Fig. 5A; this included changing the amino acid, after initiating methionine, from one that has a high frequency of acetylation (alanine or serine) to an amino acid with a low frequency of acetylation (glycine or proline). These changes were performed separately on the AAV5 VP1 and VP3 capsid proteins; in addition combined changes were made in both AAV capsid proteins. The AAV capsid protein VP2, showed no evidence of acetylation in previ- ous studies, ${ }^{29}$ thus the VP2 sequence remained unchanged from the parental sequence. AAV5 acetylation variants were analyzed by LC/MS to confirm acetylation status and the results are shown in Fig. 5A. Irrespective of the amino acid change, that is, glycine or proline, all AAV5 acetylation mutants were confirmed to have reduced acetylation. LC/MS analysis confirmed the correct molecular weights for each AAV5 capsid protein mutants. No acetylation was observed in the S-P mutants while $10 \%$ acetylation was observed in $\mathrm{S}-\mathrm{G}$ mutants. Notably, the acetylation mutants showed equivalent packaging efficiencies, compared with wild-type AAV5, suggesting that the novel amino acid changes had not adversely affected AAV vector production, or capsid protein ratios (Fig. 5B).

Next, the transduction efficiencies of the AAV5 acetylation variants were compared with wild-type AAV5, 
A

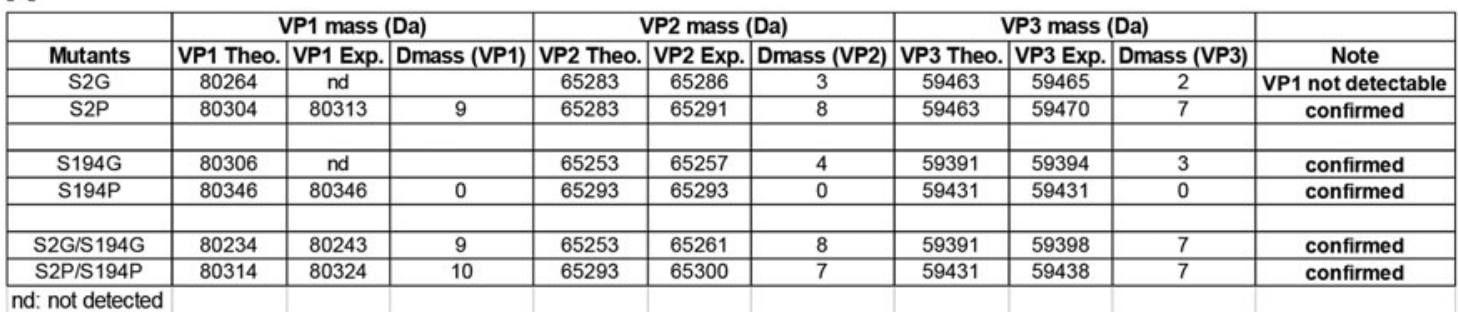

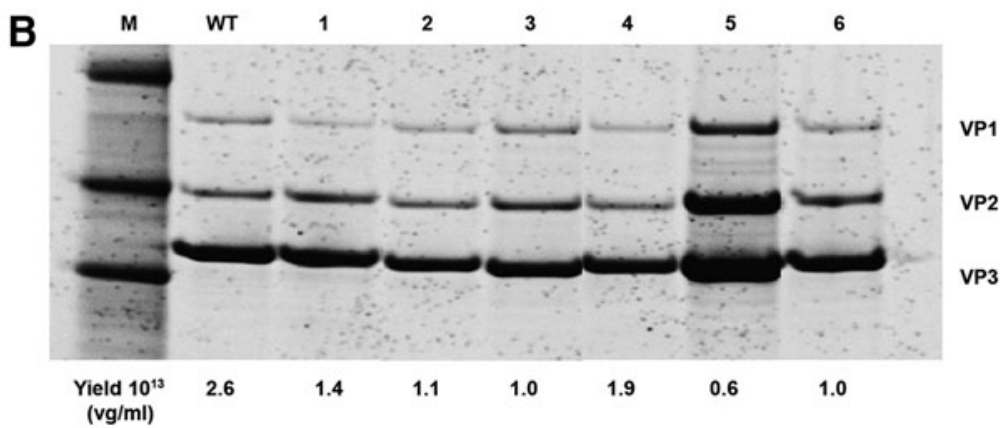

Figure 5. Characterization of AAV5 acetylation mutants by LC/MS and SDS-PAGE maintain WT levels of capsid protein expression and vector yields. (A) LC/ MS analysis to confirm the masses of VP1, VP2, and VP3 in AAV5 acetylation mutants (VP1 of AAV5S2G and AAV5S194G was not detected by mass spectrometer due to incomplete chromatographic separation of the capsid proteins). (B) SDS-PAGE gel analysis of $1 \times 10^{10}$ AAV5 acetylation capsid mutant vgs, followed by SYPR0 Red staining; lanes 1-6 represent AAV5 S2G, S2P, S194G, S194P, S2G/S194G (copurified with empty particles), S2P/S194P; VP1, VP2, and VP3 AAV capsid proteins are labeled. Lane 5 shows copurification of empty AAV particles. Vector yields for parental AAV5 and AAV5 acetylation variants are expressed as vector genomes $/ \mathrm{mL}(\mathrm{vg} / \mathrm{mL})$. LC/MS, liquid chromatography/mass spectrometry; SDS-PAGE, sodium dodecyl sulfate-polyacrylamide gel electrophoresis; VP, viral protein; WT, wild type.

in vivo, following subretinal injection to the mouse. Wildtype mice were injected with $1 \times 10^{9}$ vgs AAV5-CBAeGFP or one of each of the AAV5 acetylation mutants, harboring the same CBA-eGFP expression cassette. As previously shown (Fig. 4A), subretinal injection of AAV5eGFP resulted in robust eGFP expression in the outer retina (Fig. 6A). In contrast, the acetylation mutants AAV5S2G, AAV5S2P, AAV5S194P, AAV5S2G/S194G, and AAV5S2P/S194P showed reduced levels of eGFP expression in the retina, as evidenced by eGFP fluorescence of injected retinas (Fig. 6A) and eGFP protein levels (ELISA) (Fig. 6B). Notably, the acetylation mutant AAV5S194G-eGFP, showed a dramatic increase in eGFP expression in photoreceptor cells compared with parental AAV5-eGFP (Fig. 6A). This did not translate to a statistically different increase in eGFP protein expression, as measured by ELISA (Fig. 6B), so the performance of the AAV5S194G variant at different dose levels was explored (Fig. 7). Analysis of the vg copy number in transduced retina, revealed little difference between groups for the acetylation variants compared with parental AAV5 eGFP vector in treated retinas (Fig. 6C), at a vector dose of $1 \times 10^{9}$ vgs.

The transduction properties of the acetylation mutants, AAV5-S194G-eGFP and AAV5-S194P-eGFP, were further evaluated in a dose-response study and their performance compared with AAV5-eGFP. Increasing doses,
$1 \times 10^{8}-1 \times 10^{9} \quad$ vgs of AAV5-S194G-eGFP, AAV5S194P-eGFP, or AAV5-eGFP, were administered subretinally to a wild-type mouse. At a dose level of $1 \times 10^{9}$ vgs, the AAV5 vector showed robust transduction of photoreceptor cells (Fig. 7A); in contrast the acetylated mutant AAV5S194P, as seen in the previous experiment (Fig. 6), showed a dramatic reduction in expression compared with AAV5, whereas the AAV5-S194G variant, demonstrated a significant increase in photoreceptor cell transduction (Fig. 7A). At lower doses of vector, $5 \times 10^{8}$ and $1 \times 10^{8}$ vgs, there was a marked decrease in eGFP expression in the retinas that had received the AAV5-eGFP vector, whereas the retinas transduced with AAV5-S194GeGFP had robust eGFP expression in photoreceptors; even at doses as low as $1 \times 10^{8}$ vgs. Notably, AAV5S194P-eGFP showed little transduction of photoreceptors at all doses evaluated (Fig. 7A). The eGFP protein levels were confirmed to be statistically higher, by ELISA, in retinas treated with the AAV5S194G-eGFP compared with AAV5-eGFP at, at a dose of either $5 \times 10^{8}$ or $1 \times 10^{8} \mathrm{vgs}$. The difference in expression between the AAV5 acetylation variants and the wild-type AAV5 did not reach statistical significance at a dose of $1 \times 10^{9} \mathrm{vgs}$, presumably because of saturation of eGFP expression at this higher vector dose (Fig. 7B), and as seen in the previous experiment, study (Fig. 6B). Analysis of vg copy number revealed a dose-response for both vectors, retinas transduced 
A
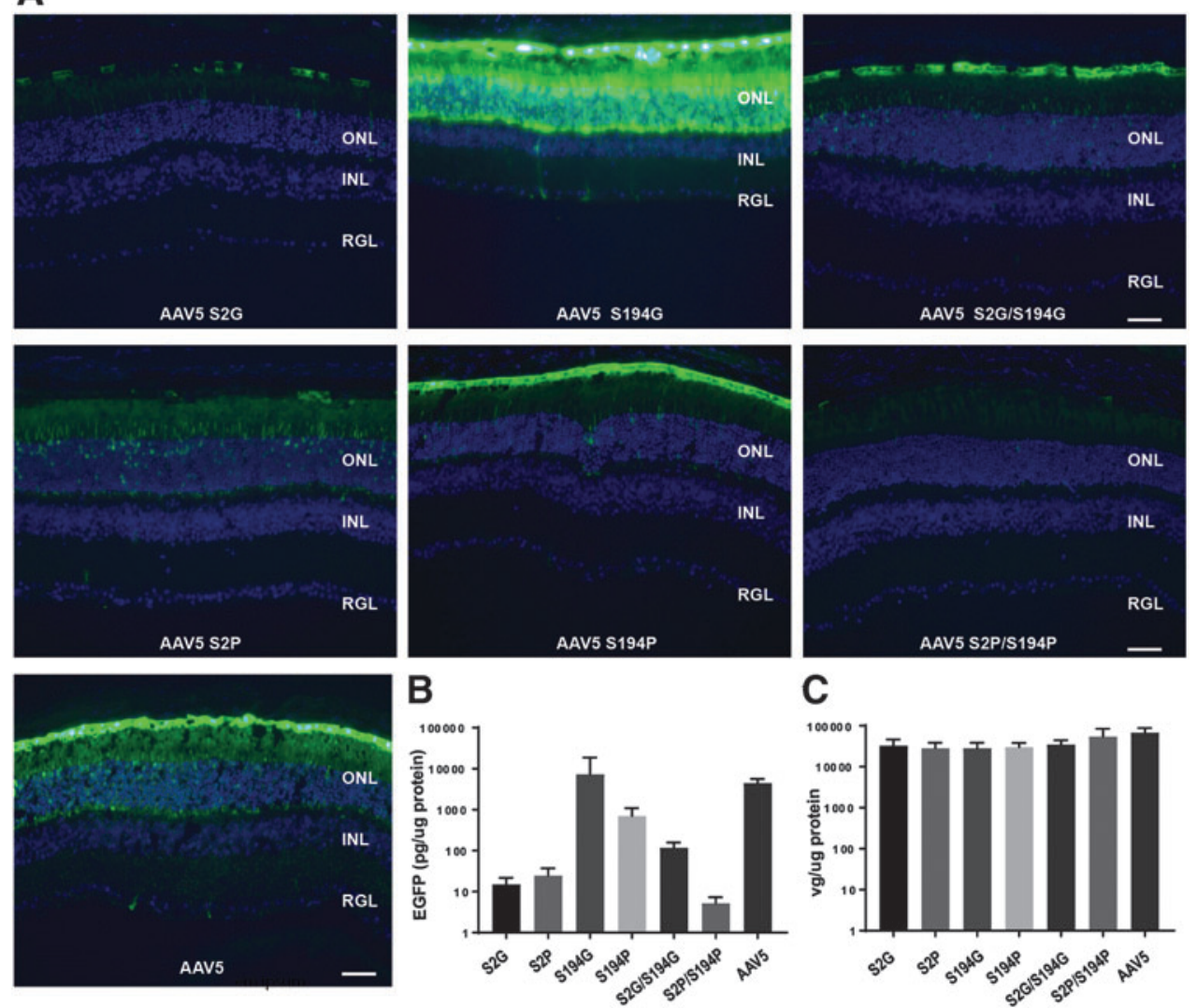

Figure 6. Effect of AAV5 acetylation on photoreceptor transduction. (A) Comparison of photoreceptor transduction efficiency between AAV5 and AAV5 acetylation variants (AAV5S2G, AAV5S194G, AAV5S2G/S194G, AAV5S2P, AAV5S194P, and AAV5S2P/S194P). WT mice $(n=10)$ were injected with the same vg copies of AAV5 and AAV5 acetylation variants and eyes were collected 4 weeks after injection and processed for cryosectioning. Fluorescence microscopy analysis showed strong eGFP expression in the ONL of AAV5S194G injected retinas as compared with its parental AAV5 and other variants. Scale bar $50 \mu \mathrm{m}$. (B) Quantification of eGFP by ELISA of retinas from C57BL/6 mice $(n=10)$, 4 weeks following subretinal injection of AAV5 and AAV5 acetylation variants encoding for eGFP. Error bars represent \pm SD. (C) qPCR analysis of AAV vg copies in transduced mouse retinas $(n=10)$ following subretinal injections of AAV5 and AAV5 acetylation variants. Error bars represent \pm SD. ELISA, enzyme-linked immunosorbent assay; qPCR, quantitative polymerase chain reaction; SD, standard deviation.

with the AAV5S194G-eGFP acetylation variant, at a dose of $5 \times 10^{8}$ or $1 \times 10^{8} \mathrm{vgs}$, had higher vg copies, compared with retinas treated with AAV5-eGFP at a similar dose (Fig. 7C).

\section{Evaluation of AAV5 capsid variants in NHP retinal explants}

We evaluated the use of an ex vivo NHP organotypic explant system to test the transduction efficiency and the tropism of our novel variants and to assess translational fidelity of AAV variants between species. To that end, retinal explants from NHPs were established and the transduction efficiency of the acetylation variants AAV5S194G \& AAV5S194P was evaluated. Seven days after plating, the cultured retinas retained normal architecture, including intact rod and cone photoreceptor inner and outer segments, a full ONL, and outer to inner retinal connectivity (Fig. 8A). The transduction efficiency of the AAV5 and AAV5 acetylation variants in the retinal explants mimicked the transduction efficiency observed in the mouse retina (Fig. 6A), and for unmodified AAV5, mimicked the transduction activity also seen in the NHP retina (Fig. 3A).
Confirming what was seen in the mouse retina (Fig. 7), the AAV5S194G variant showed superior transduction efficiency in the NHP ONL compared with the AAV5 parental vector (Fig. 8A, C). In contrast, compared with the parental AAV5, the AAV5S194P variant revealed decreased transduction efficiency in the NHP retinal explant, similar to its performance in the mouse retina (Fig. 7). The authenticity of the organotypic culture was confirmed by the performance of the AAV5-eGFP parental vector, which demonstrated robust transduction of the ONL (Fig. 8A, C), similar to the transduction performance seen with subretinal delivery of AAV5-eGFP vector to the NHP retina (Fig. 3A). Finally, evaluation of the acetylation mutants in the NHP retinal explant model revealed an additional benefit to the AAV5S194G variant over unmodified AAV5. This capsid variant selectively transduces photoreceptor cells, with little transduction observed in other cell types (Fig. 8A, C). The eGFP protein levels in the NHP retinal explant cultures were quantified by ELISA (Fig. 8B). There was no difference in eGFP expression between the AAV5 and AAV5S194G 

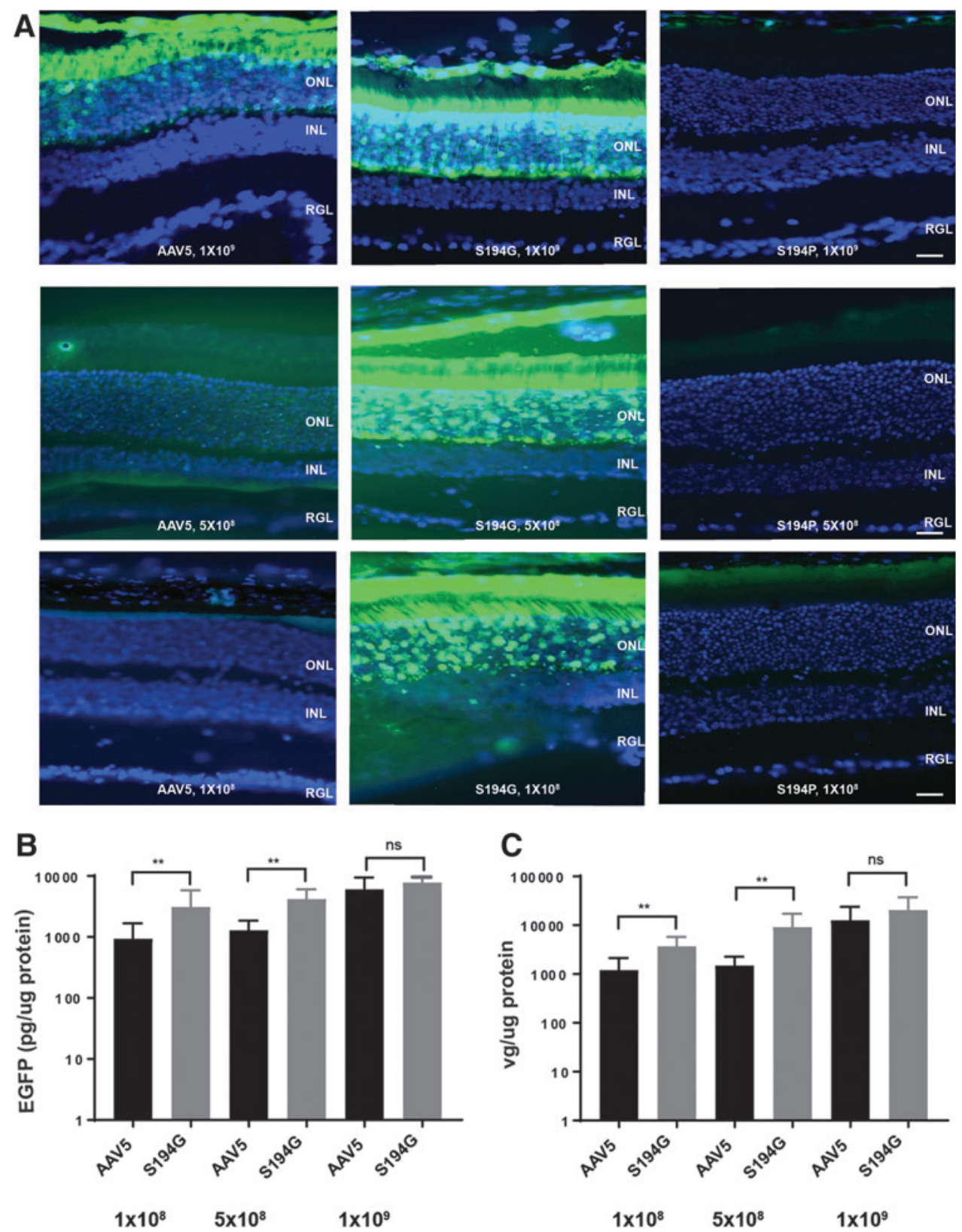

Figure 7. Comparison of eGFP expression after subretinal delivery of AAV5 acetylation variants in a dose-dependent manner. (A) Representative fluorescent images of retinal cryosections at 4 weeks postinjection showing transduction comparison (native eGFP expression shown in green) in eyes injected with AAV5, AAV5 S194G, AAV5 S194P at different doses (low $1 \times 10^{8}$, medium $5 \times 10^{8}$, and high $1 \times 10^{9}$ ). Nuclei is stained with DAPI (blue). Scale bar $50 \mu \mathrm{m}$. (B) Quantification of eGFP protein by ELISA of retinas from C57BL/6 mice $(n=12), 4$ weeks following subretinal injection of AAV5 and AAV 5 acetylation variants encoding for eGFP. **Indicates that the value is statistically significant at $p<0.01$, ns, respectively, compared with AAV5 injected retinas, according to the twotailed unpaired $t$-test. Error bars represent \pm SD. (C) qPCR analysis of AAV vg copies in transduced retinas $(n=12)$ following subretinal injections of AAV5 or AAV5 acetylation variants expressing eGFP. ${ }^{*}$ Indicates that the value is statistically significant at $p<0.01$, ns, respectively, compared with AAV5-injected retinas, according to the two-tailed unpaired $t$-test. Error bars represent \pm SD. DAPI, 4',6-diamidino-2-phenylindole; ns, not significant.

capsid. The eGFP ELISA quantifies the total GFP protein in all cell types in the retinal explant culture and as a result does not highlight the differences in tropism between these two capsids observed at the cellular level in the fluorescence images (Fig. 8C). There was no detectable eGFP in the NHP retinal explants infected with the AAV5S194P vector (Fig. 8B).

\section{Evaluating the role of deamidation on AAV2 transduction in the retina}

Analysis of quality attributes of AAV vectors generated using the PCL production platform, including PTMs on the AAV capsid proteins, revealed several interesting observations. Specifically, the PCL process, in the context of AAV2 vectors, resulted in AAV vector preparations 

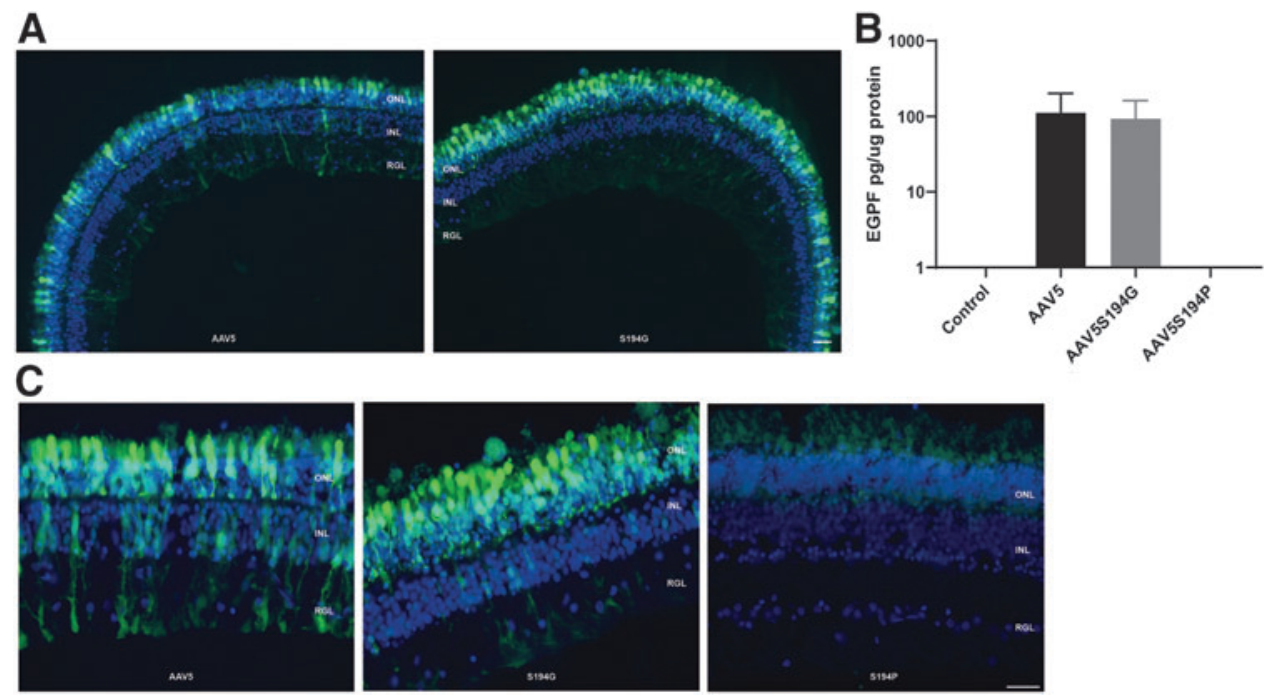

Figure 8. Comparison of native eGFP fluorescence following ex vivo administration of AAV5 and AAV5 acetylation variants in NHP retinal tissue. (A) Neural retinal tissue was obtained from postmortem NHP eyes. Biopsy punches were made and were cultured on membranes in six-well plates and transduced with different AAV variants encoding eGFP. The tissues were harvested 6 days posttransduction, fixed and imaged for native eGFP expression. The potency of AAV serotypes was compared by analyzing transduction of cell types in each of the ONL, INL, and RGL. AAV5 variant AAV5S194G showed higher potency in transduction of photoreceptors than native AAV5. Scale bar $20 \mu \mathrm{m}$. (B) Quantification of eGFP by ELISA of NHP retinal explants $(n=2) 5$ days following infection with AAV5 eGFP, AAV5S194G eGFP, and AAV5S194P eGFP. AAV vectors at a dose of $1.8 \times 10^{11}$ vgs was used for each explant. (C) Higher magnification images showing the expression GFP following AAV transduction. Interestingly, the eGFP expression was predominantly observed in ONL with AAV5 S194G while eGFP expression was observed in all layers with AAV5. Scale bar $50 \mu \mathrm{m}$.

that consistently had a protein running below VP1, as compared with a similar AAV2 vector produced through the triple transfection production platform (Fig. 9B). LC/ MS analysis revealed the protein to be a truncated form of VP1 protein (tVP1), lacking the first 34 amino acids, with acetylated A35 confirmed to be the N-terminal amino acid (article in preparation). Deamidation, in which side chain amide groups, typically asparagine, are converted to aspartic acid, is a common PTM, which often signals a protein for degradation. We hypothesized that tVP1 was a result of deamidation at a neighboring asparagine, N57, resulting in the proteolytic cleavage of VP1 at A35 to generate tVP1 (Fig. 9A). LC/MS analysis confirmed the deamidation status of N57, in the context of the AAV2 PCL-derived vector, to be higher at $18.4 \%$, compared with $6.7 \%$, for a comparable AAV2 vector generated by triple transfection (Fig. 9D). Notably, there was no measured difference in deamidation at other potential deamidation sites in the AAV2 capsid sequence, including N511G or N717G. Interestingly, the infectivity of the AAV2 PCL vector trended lower in an analytical in vitro assay (Fig. 9D), thus deamidation mutants were generated to further explore the role of deamidation on AAV2 infectivity in the retina. To that end, the N57 deamidation site was mutated to aspartic acid, N57D, to generate a capsid that would have complete deamidation at that residue. Additionally, variant G58D was generated to control for the effects of introducing an aspartic acid in this region of the AAV2 capsid sequence. AAV2N57D-eGFP and AAV2G58D-eGFP variants were generated and produced using the triple transfection production method. The AAV2 variants had similar packaging efficiencies and capsid protein profiles (Fig. 9C, E), as the parental AAV2 capsid. LC/MS analysis of the deamidation variants confirmed that the AAV2G58D-eGFP was $1.1 \%$ deamidated (to become aspartic acid), wild-type AAV2-eGFP was $5.7 \%$ deamidated, whereas the AAV2N57D-eGFP was $100 \%$ deamidated (Fig. 9E).

The deamidation variants were next evaluated in vivo to assess the role of this PTM on AAV2 transduction activity. Intravitreal delivery of AAV2-eGFP vector to wild-type mice resulted in significant transduction of retinal ganglion cells (Fig. 10C). A similar result was observed with the AAV2G58D-eGFP variant, in contrast, intravitreal delivery of AAV2N57D-eGFP resulted in poor retinal transduction as measured by eGFP fluorescence (Fig. 10C) or ELISA (Fig. 10A). The vg copies of retinal tissue transduced with the AAV2N57D-eGFP variant trended lower than the levels measured with unmodified AAV2eGFP or AAV2G58D-eGFP, suggesting a possible correlation to the reduced transgene expression measured with the AAV2N57D-eGFP variant (Fig. 10B).

\section{DISCUSSION}

We have shown, using a rational design approach and knowledge of AAV receptor binding, surface charge, and capsid protein PTMs, that AAV variants with novel tropism and transduction for retina and cornea can be generated (Table 2). Previously, we reported on the 


\section{AAV2}

(1) MAADGYLPDWLEDTLSEG IRQWWKLKPGPPPP

B
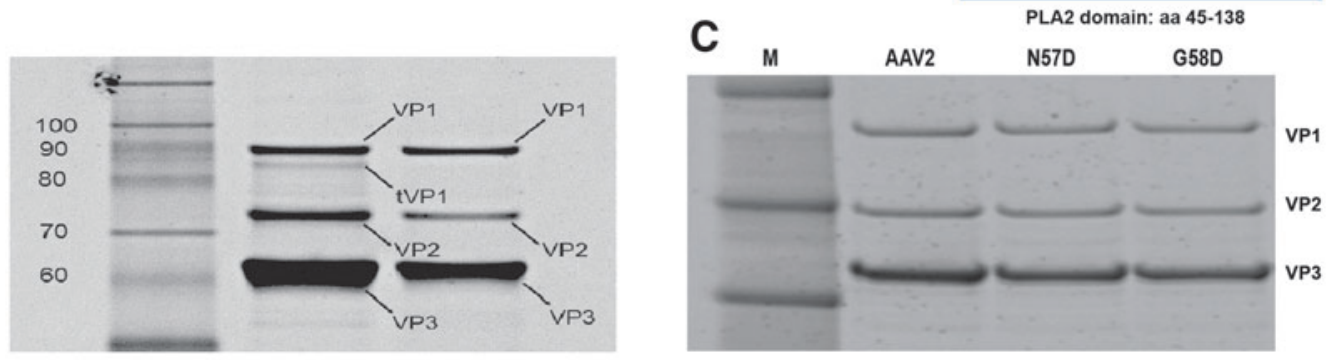

D

\begin{tabular}{|c|c|c|c|c|c|c|}
\hline \multirow{2}{*}{ Vector } & \multirow{2}{*}{$\begin{array}{c}\text { Production } \\
\text { Platform }\end{array}$} & Potency & Infectivity & \multicolumn{3}{|c|}{$\begin{array}{c}\text { \% Deamidation at 3 } \\
\text { different N(G) sites }\end{array}$} \\
\cline { 3 - 7 } & & TU : IU & VG : IU & N67 & N611 & N717 \\
\hline \multirow{2}{*}{ AAV2eGFP } & $\mathrm{TTX}$ & 24 & 6 & 6.7 & 39.6 & 27.4 \\
\cline { 2 - 7 } & $\mathrm{PCL}$ & 97 & 19 & 18.4 & 42.3 & 28 \\
\hline
\end{tabular}

E

\begin{tabular}{|c|c|c|c|}
\hline & AAV2 & N57D & G58D \\
\hline \% Deamidation & 5.7 & 100 & 1.1 \\
\hline Yield $(\mathrm{vg} / \mathrm{ml})$ & $8.7 \times 10^{12}$ & $5.46 \times 10^{12}$ & $3.6 \times 10^{12}$ \\
\hline
\end{tabular}

Figure 9. Altering deamidation levels within the PLA2 domain of AAV2 VP1 does not impact capsid protein expression or vector yields but impacts potency. (A) Protein sequence motifs located within the VP1 N-terminus of AAV2. A35 is the N-terminal amino acid for tVP1. The 57NG58 is a canonical deamidation motif located within the PLA2, domain. (B) SDS-PAGE analysis of $1 \times 10^{10}$ AAV2 vector generated by either TTx or PCL production method, followed by SYPRO Red staining. Lane 1: Molecular Weight Markers. Lane 2: AAV2 vector generated by the PCL method. Lane 3: AAV2 generated by the TTx. tVP1 represents a truncated VP1 protein. (C) SDS-PAGE gel analysis of $1 \times 10^{10} \mathrm{vgs}$ of AAV2 and AAV2 deamidation variants, N57D and G58D, followed by SYPRO Red staining. (D) Characterization of AAV2 vectors generated by TTx and PCL platforms. In vitro potency is represented by particle:infectivity ratio for each AAV vector preparation. The difference in \% deamidation at potential NG sites in the AAV2 capsid is reported for AAV2 vectors generated using either the TTx or the PCL production platform. Percentage values for deamidation were determined by LC-MS. (E) Characterization of AAV2 and AAV2 deamidation variants. Vector yields were determined by qPCR and are represented as $\mathrm{vg} / \mathrm{mL}$. The $\%$ deamidation of parental AAV2, AAV2 N57D, and AAV2 G58D deamidation variants was determined by LC/MS. PCL, producer cell line; PLA2, phospholipase A2; TTx, triple transfection production method.
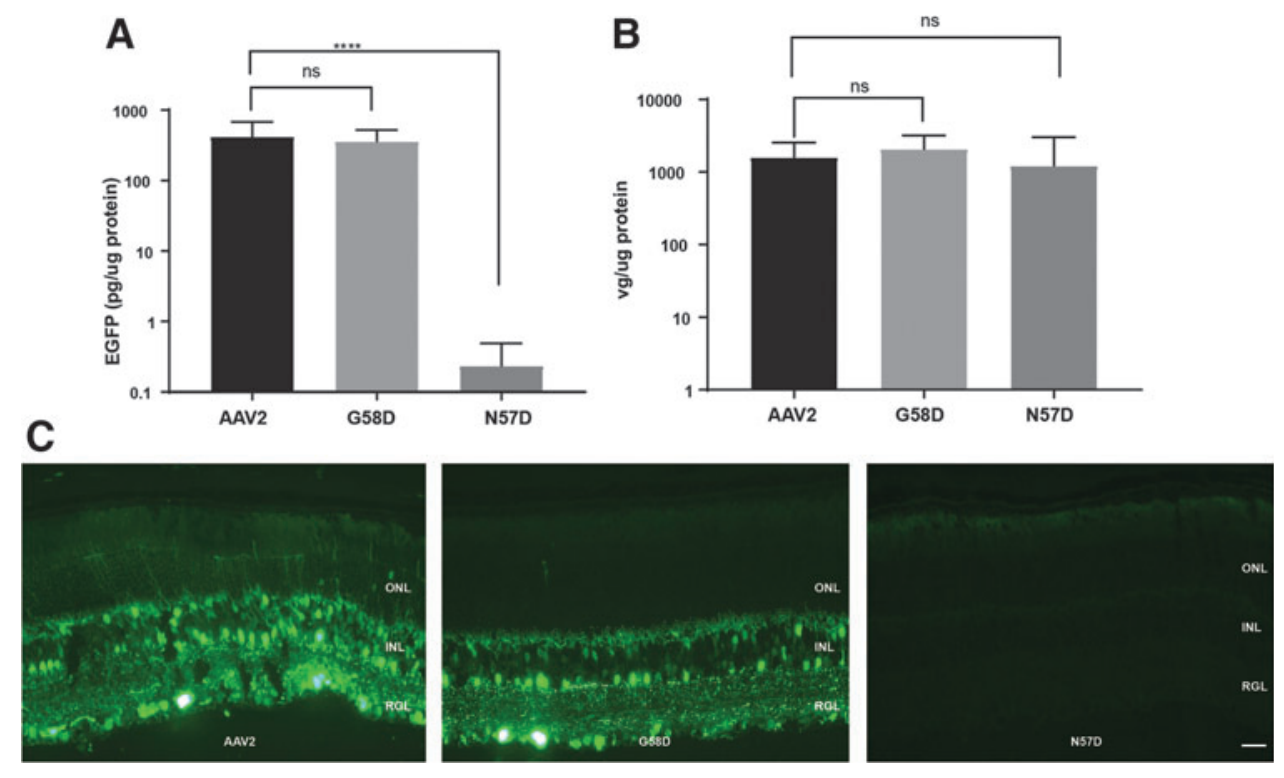

Figure 10. Effect of AAV2 capsid deamidation on retinal transduction. (A) Quantification of eGFP by ELISA of retinas from WT mice ( $n=10) 4$ weeks following intravitreal injection of AAV2 and AAV2 deamidation variants encoding eGFP. ${ }^{* * *}$ Indicates that the value is statistically significant at $p<0.0001$ compared with AAV2-injected retinas, according to the two-tailed unpaired $t$-test. Error bars represent \pm SD. (B) qPCR analysis of AAV genome copies in transduced retinas $(n=10)$, following intravitreal injections of AAV2 and AAV2 deamidation variants. ns according to the two-tailed unpaired $t$-test. Error bars represent \pm SD. (C) Fluorescence analysis showing native eGFP expression patterns in WT mice after intravitreal injection of AAV2 and AAV2 deamidation mutants. Scale bar $50 \mu \mathrm{m}$. ns, not statistically significant. 
Table 2. Summary of adeno-associated viral capsid variants generated for these studies

\begin{tabular}{llll}
\hline Variant & Delivery & Novel Transduction Phenotype & \\
\hline AAV2-HBKO & SR & Enhanced photoreceptor & Mechanism \\
AAV5-G474R & IVIT & Corneal endothelium & Ultered receptor binding \\
AAV5-N564R & IVIT & Corneal endothelium & Unknown-altered receptor binding? \\
AAV5-N573R & IVIT & Corneal endothelium & Unknown-altered receptor binding? \\
AAV5-S2G & SR & Decreased photoreceptor & Deacetylated VP1 \\
AAV5-S2P & SR & Decreased photoreceptor & Deacetylated VP1 \\
AAV5-S194G & SR & Increased photoreceptor & Deacetylated VP3 with the substitution of a flexible glycine residue \\
AAV5-S194P & SR & Decreased photoreceptor & Deacetylated VP3 with the substitution of a bulky nonflexible proline residue \\
AAV5-S2G/S194G & SR & Decreased photoreceptor & Deacetylated VP1/VP3 \\
AAV5-S2P/S194P & SR & Decreased photoreceptor & Deacetylated VP1/VP3 \\
AAV2-N57D & IVIT & Noninfectious & Increased VP1 deamidation \\
AAV2-G58D & IVIT & None observed & Decreased VP1 deamidation \\
\hline
\end{tabular}

IVIT, intravitreal delivery; VP, viral protein; SR, subretinal.

improved transduction performance of our AAV2-HBKO variant in the context of the mouse retina and central nervous system (CNS). ${ }^{20}$ The AAV2-HBKO variant has key surface arginines mutated in regions of the capsid that facilitate the binding of AAV2 to its cognate receptor, HSPG. This heparan-binding knockout variant demonstrated novel transduction patterns in the mouse CNS and retina, ${ }^{20}$ and in follow-up studies, the novel CNS transduction properties were confirmed to translate to the NHP brain. ${ }^{46}$ However, the translatability of the transduction performance of the AAV2-HBKO variant from mouse to NHP retina was not confirmed. In this study, we show that the AAV2-HBKO variant exhibited high levels of transduction and transgene expression to the outer retina, when the identical vector was delivered subretinally to either mice or NHP. Previously, subretinal delivery of AAV2 vectors to the NHP retina demonstrated limited photoreceptor cell transduction, with most of the transgene expression restricted to the RPE; this was demonstrated in the context of a ubiquitous CBA promoter-driving expression of a human RPE65 transgene. ${ }^{47}$ Thus, the ablation of HS binding of AAV2-based vectors, as shown here, is an effective strategy for enhancing biodistribution to primate photoreceptors. Further restriction of transgene expression to rod photoreceptors was achieved with the use of a rhodopsin promoter. Rod photoreceptor transduction was observed across the area of the subretinal bleb, with transduction dramatically decreasing in the cone-rich area of the fovea. In the NHP retinal study, the transduction profile of the AAV2-HBKO variant was compared with that of an AAV5 vector harboring the identical eGFP expression cassette, and although the extent of rod photoreceptor transduction was equivalent for both vectors, notably in the NHP eye, the AAV2-HBKO vector demonstrated the ability to spread past the border of the subretinal bleb. This was confirmed by both in vivo imaging and postmortem histology.

It can be hypothesized that the improved transduction and spread observed with the AAV2-HBKO variant, is partially because this variant is not sequestered by HSPG and can traffic beyond the borders of the bleb. One caveat to the interpretation of the transduction activity of the AAV2-HBKO in the NHP retina, is that the $n$ value (4) is limited, and a more comprehensive dose-ranging study with a larger $n$ value is needed to confirm these preliminary observations. Altogether, these results establish that the ablation of HS binding of AAV2-based vectors may be an effective strategy for enhancing biodistribution to photoreceptors and promoting vector spread in the primate retina, a valuable attribute when treating inherited retinal diseases that require a more global correction of the retina.

The AAV2-HBKO variant revealed the importance of arginines, and by extension capsid surface charge, on transduction activity in the retina. The AAV2-HBKO vector has two positively charged arginine residues (R585A and R588A) substituted for two hydrophobic alanine residues on the surface of the AAV2 capsid. These substitutions significantly altered the tropism of AAV2 in the retina. In contrast to parental AAV2, AAV2-HBKO lacks tropism for inner retina following an intravitreal administration, ${ }^{20}$ whereas it acquires a novel tropism for photoreceptors following subretinal delivery.

One hypothesis to explain the different tropism AAV2HBKO demonstrated with intravitreal delivery is that, AAV2 surface arginines are important for navigating the proteoglycan-rich region of the inner limiting membrane, a barrier separating the vitreous from the neural retina. ${ }^{35}$ We questioned if adding arginines could impact the tropism and transduction activity of a different capsid, AAV5, which has relatively few surface arginines compared with AAV2. It is known that while subretinally delivered AAV5 demonstrates superior photoreceptor transduction (current study and Ref. ${ }^{45}$ ), its transduction activity is limited following intravitreal surgery. ${ }^{48}$ Thus, the effect of altering surface charge on the AAV5 capsid was explored to determine if improved transduction activity in the retina could be achieved, both in the context of intravitreal and subretinal delivery. Intravitreal delivery of AAV5 arginine variants, AAV5G474R, AAV5N564R, and AAV5N573R, to the mouse resulted in limited retinal 
transduction, comparable to the pattern observed following intravitreal delivery of the AAV5 parental capsid. However, in contrast to the AAV5 parental capsid, all AAV5 arginine variants acquired a novel tropism for and improved transduction activity in corneal endothelial cells. This is the first report of an AAV vector that can transduce corneal endothelial cells following a noninvasive procedure such as intravitreal surgery. Serotypes, such as AAVAnc80L65 $65^{5}$ and AAV8G $9,{ }^{49}$ first-generation serotypes, AAV1-AAV9, ${ }^{50}$ and AAV2 tyrosine variants, ${ }^{6}$ have been reported to transduce corneal endothelial cells in rodents, but only following intracameral delivery, a surgical procedure that requires puncturing the cornea, with the concomitant risk of damage to the anterior chamber of the eye. The enhanced AAV5 vectors described in this study will need to be further tested to determine if the gains in transduction from the vitreous, as seen in mouse, translate to similar improvements in the primate eye. If translatability is confirmed, these novel AAV5 arginine variants show promise for the treatment of inherited corneal dystrophies or systemic diseases affecting the cornea. ${ }^{50}$

The AAV2-HBKO and AAV5 arginine variants were generated by a rational design approach using knowledge of receptor binding and surface charge, respectively. Next, additional variants were generated using knowledge gained from LC/MS analysis of the AAV capsid, where PTMs, including acetylation on AAV5VP1 (Ser2) and VP3 (Ser194) capsid proteins were revealed. ${ }^{29}$ To understand the general function of $\mathrm{N}$-terminal acetylation on AAV capsid proteins, we examined the role of $\mathrm{N}$-terminal acetylation on AAV5 transduction in the retina. Proteins in which the second amino acid is either a proline or a glycine are the most unfavorable proteins for N-terminal acetylation. ${ }^{51}$ Thus, a change in serine at position 2 in the AAV5 VP1 protein, to glycine or proline (variants AAV5S2G and AAV5S2P, respectively), resulted in reduced acetylation on VP1, as verified by LC/MS, and a concomitant reduction in mouse retinal transduction. This reduced transduction was evident when the $\mathrm{N}$-terminal acetylation of VP1 was decreased in isolation, or in combination with reduced $\mathrm{N}$-terminal acetylation in the AAV5 VP3 protein, that is, variants AAV5S2G/S194G and AAV5S2P/S194P. Notably, when VP1 N-terminal acetylation was preserved and VP3 N-terminal acetylation reduced, by changing serine 194 to a glycine (variant AAV5S194G), there was a significant improvement in retinal transduction compared with parental AAV5; the improved transduction was evident in both the mouse retina and NHP retinal explants. These data underscore the importance of VP1 Nterminal acetylation in AAV5 transduction and suggests that this PTM maybe a requirement for AAV endosomal release, a process that requires a $\mathrm{pH}$ conformational change in the VP1 N-terminus, before trafficking to the nucleus. ${ }^{27}$

$\mathrm{N}$-terminal acetylation has been shown to influence the localization of other proteins. Arl3p, an "Arf-like" (Arl)
GTPase, which is crucial for the organization of membrane traffic, requires its $\mathrm{N} \alpha$-acetyl group for correct targeting to the Golgi membrane. ${ }^{52}$ In contrast, N-terminal acetylation on VP3 is redundant, as evidenced by the improved transduction activity of the VP3 N-terminal acetylation variant AAV5S194G. However, when VP3 Nterminal acetylation was reduced by substituting S194 with the bulkier proline, the resulting variant, AAV5S194P, demonstrated limited transduction in the mouse retina and NHP retinal explant model. The reason for the difference in transduction activity between AAV5S194G and AAV5S194P variants, maybe best explained by the difference in size of the substituted residues, proline versus glycine, which in turn influences the local flexibility of the peptide backbone. Glycine is a hydrophilic amino acid that provides significant flexibility, in contrast proline is nonpolar and likely provides more rigidity to the capsid; this rigidity may impede the process of AAV5 capsid uncoating, having a negative impact on AAV5 transduction in the retina. Altogether, these data underscore the importance of maintaining the $\mathrm{N}$-terminal acetylation on VP1 for AAV5 transduction activity, additionally, N-terminal acetylation on the AAV5 VP3 protein is not necessary for transduction, but rather the flexibility of the amino acid at the second position in the VP3 N-terminus influences transduction activity by controlling capsid rigidity and likely capsid uncoating, a critical step in the AAV transduction process. This discovery highlights an area that might be further explored to improve transduction activity of other AAV serotypes.

Deamidation, in which side chain amide groups, typically asparagine, are converted to aspartic acid, is a common PTM that can signal a protein for proteolysis. ${ }^{53} \mathrm{~A}$ common attribute with AAV2 vectors generated using the PCL process, ${ }^{41,43}$ when compared with the same vector made by transient transfection, is the presence of a tVP1, and analytically, a trend for decreased in vitro potency. We hypothesized that the truncated VP1 species may have resulted from proteolysis signaled by deamidation on 57NG58 (Fig. 9A). LC/MS analysis confirmed increased 57NG58 deamidation for PCL-derived vectors, and the effects of genetically forcing deamidation on AAV transduction activity, was explored. Our AAV2N57D variant showed total loss of in vivo transduction activity, in both mouse and NHP retina. Additionally, changing N57 to either a lysine $(\mathrm{N} 57 \mathrm{~K})$ or a glutamine (N57Q), ablated in vitro transduction (Supplementary Fig. S4). Surprisingly, mutating the neighboring glycine to an aspartate (variant G58D), had no effect on AAV2 transduction activity, suggesting that the G58 residue is tolerant of change, in the context of AAV2 function in the mouse eye. Altogether, these data underscore the importance of the N57 residue in AAV2 transduction activity, and suggest that changes in N57, either by deamidation or directed mutagenesis, result in functional loss. 
VP1/VP2 protein N-terminal sequences harbor infection-relevant functional domains, such as a PLA2 catalytic subunit. ${ }^{25,26,28}$ Importantly, the N57 residue lies in the calcium-binding loop of the PLA2 domain, suggesting a possible role for N57 in binding this essential cofactor of enzyme activity and viral endosomal escape, an obligatory step in AAV2 infection. ${ }^{26}$ Alternatively, maintaining N57 at the VP1 N-termini is critical for the correct unfolding and surface exposure of the PLA2 domain. ${ }^{10,27}$ Data to support the hypothesis that the defect in the AAV2N57D mutant is likely endosomal escape or uncoating are supported by the fact that there are measurable vgs in the cell, but no detectable transgene expression (Fig. 9). In summary, our data suggest that strategies to rationally design AAV2 vectors for enhanced transduction in the retina should avoid any changes in the N57 VP1 N-terminal residue, whereas changes in neighboring residues, that is, G58, are well tolerated, and suggest that $\mathrm{a}+1$ site mutagenesis strategy may be used to stabilize 57NG58 asparagine from inadvertent deamidation. Interestingly, during our studies, Giles et al. ${ }^{54}$ reported on NG deamidation-induced functional loss for AAV8, and evaluated strategies to stabilize amides to improve vector performance. The authors also highlighted the importance of controlling for deamidation during AAV manufacturing and recommended quantitating the level of deamidation as a critical quality attribute for clinical AAV vector lot release. ${ }^{54}$

The current study describes the optimization of an NHP retinal organotypic explant system that facilitated rapid assessment of the transduction activity of our novel variants in a retina from a species other than mice. The lack of translation from mouse to NHP brain has been reported for the AAVPHP.B variant, ${ }^{55}$ so with this precedent, we wanted to confirm if the improved transduction activity of AAV5S194G in mouse retina was translated to NHP photoreceptors. The fidelity of the NHP transplant model was confirmed using AAV5, and the superior transduction activity of the AAV5S194G variant in the explant model was demonstrated. Notably, evaluation of the AAV5S194G variant in the NHP retinal model revealed an additional benefit to this capsid; AAV5S194G transduction is predominantly in primate photoreceptors, in contrast parental AAV5 transduces photoreceptors, INL and GCL. One caveat to the NHP explant model is that it does not provide data relating to potential immunological findings for the novel AAV capsids, however, the model provides insights to the potential translatability of an AAV capsid for the treatment of human retinal disease.

In conclusion, this study underscores the utility of a rational design approach to engineer capsids for targeting and improving the efficacy of AAV vectors for ocular gene therapy. Prior knowledge of AAV receptor binding, surface charge, and PTMs was applied to the design of optimized capsids that provide improved retinal spread, a novel corneal cell tropism and increased photoreceptor transduction, respectively. Importantly, the attributes provided by these novel capsids will add to the efficacy, specificity, and safety of their potential use in gene therapy for a wide variety of human ocular diseases.

\section{ACKNOWLEDGMENTS}

The authors would like to acknowledge Bindu Nambiar, Shelley Nass, Maryellen Mattingly and Denise Woodcock from the Gene Therapy Research Vector Core and Rachel Diangelo and Simon Godwin from the Gene Therapy Development and Manufacturing group.

\section{AUTHOR DISCLOSURE}

All authors are employees of Sanofi.

\section{FUNDING INFORMATION}

No funding was received for this article.

\section{SUPPLEMENTARY MATERIAL}
Supplementary Figure S1
Supplementary Figure S2
Supplementary Figure S3
Supplementary Figure S4
Supplementary Table S1

\section{REFERENCES}

1. Dias MF, Joo K, Kemp JA, et al. Molecular genetics and emerging therapies for retinitis pigmentosa: basic research and clinical perspectives. Prog Retin Eye Res 2018;63:107-131.

2. Shahryari A, Jazi MS, Mohammadi S, et al. Development and clinical translation of approved gene therapy products for genetic disorders. Front Genet 2019;10:1-25.

3. Dalkara D, Byrne LC, Klimczak RR, et al. In vivo directed evolution of a new adeno-associated virus for therapeutic outer retinal gene delivery from the vitreous. Sci Transl Med 2013:5:76-87.
4. Ye GJ, Budzynski E, Sonnentag P, et al. Safety and biodistribution evaluation in cynomolgus macaques of rAAV2tYF-CB-hRS1, a recombinant adeno associated virus vector expressing retinoschisin. Hum Gene Ther Clin Dev 2015;26:165-172.

5. Wang L, Xiao R, Andres-Mateos E, et al. Single stranded adeno-associated virus achieves efficient gene transfer to anterior segment in the mouse eye. PLoS One 2017;12:1-12.

6. Bogner B, Boye, SL, Min SH, et al. Capsid mutated adeno-associated virus delivered to the anterior chamber results in efficient transduction of tra- becular meshwork in mouse and rat. PLoS One 2015;10:1-16

7. Maclachlan TK, Lukason M, Collins $M$, et al. Preclinical safety evaluation of AAV2-sFLT01-a gene therapy for age-related macular degeneration. Mol Ther 2011;19:326-334.

8. Büning H, Srivastava A. Capsid modifications for targeting and improving the efficacy of AAV vectors. Mol Ther Meth Clin D 2019;12:248-265.

9. Herrmann AKM, Bender C, Kienle E, et al. A robust and all-Inclusive pipeline for shuffling of adenoassociated viruses. ACS Synth Biol 2019;8:194-206. 
10. Balasubramanian V, Yarbrough J, Domsic J, et al. Structure and dynamics of adeno-associated virus serotype 1 VP1 unique $\mathrm{N}$-terminal domain and its role in capsid trafficking. J Virol 2013;87:49744984.

11. Xie Q, Bu W, Bhatia S, et al. The atomic structure of adeno-associated virus (AAV-2), a vector for human gene therapy. PNAS 2002;99:1040510410.

12. Lerch T, Xie Q, Chapman, MS. The structure of adeno-associated virus serotype 3B (AAV-3B): insights into receptor binding and immune evasion. Virology 2010;403:26-36.

13. Padron E, Bowman V, Kaludov N, et al. Structure of adeno-associated virus type 4. J Virol 2005;79: 5047-5058

14. Govindasamy L, DiMattia MA, Gurda BL et al. Structural insights into adeno-associated virus serotype 5. J Virol 2013;87:11187-11199.

15. Ng R, Govindasamy L, Gurda BL, et al. Structural characterization of the dual glycan binding adenoassociated virus serotype 6. J Virol 2010;84: 12945-12957.

16. Nam HJ, Lane MD, Padron E, et al. Structure of adeno-associated virus serotype 8 , a gene therapy vector. J Virol 2007:81:12260-12271.

17. Kern A, Schmidt K, Leder C, et al. Identification of a heparin-binding motif on adeno-associated virus type 2 capsids. J Virol 2003;77:11072-11108.

18. Kaludov N, Brown KE, Walters RW, et al. Adenoassociated virus serotype 4 (AAV4) and AAV5 both require sialic acid binding for hemagglutination and efficient transduction but differ in sialic acid linkage specificity. J Virol 2001;75:6884-6893.

19. Di Pasquale G, Davidson BL, Stein CS, et al. Identification of PDGFR as a receptor for AAV-5 transduction. Nat Med 2003;9:1306-1312.

20. Sullivan JA, Stanek LM, Lukason MJ, et al. Rationally designed AAV2 and AAVrh8R capsids provide improved transduction in the retina and brain. Gen Ther 2018;25:205-219.

21. Boye SL, Bennett A, Scalabrino M, et al. Impact of heparan sulfate binding on transduction of retina by recombinant adeno-associated virus vectors. J Virol 2016:90:4215-4231.

22. Lubeck MD, Lee HM, Hoggan MD, et al. Adenovirus-associated virus structural protein sequence homology. J Gen Virol 1979;45:209216.

23. McPherson RA, Rose JA. Structural proteins of adenovirus-associated virus: subspecies and their relatedness. J Virol 1982:46:523-529.

24. Becerra SP, Rose JA, Hardy M, et al. Direct mapping of adeno-associated virus capsid proteins $B$ and $C$ : a possible ACG initiation codon. PNAS 1985;82:7919-7923.

25. Girod A, Wobus CE, Za!dori Z, et al. The VP1 capsid protein of adeno-associated virus type is carrying a phospholipase A2 domain required for virus infectivity. J Gen Virol 2002;83:973-978.
26. Zadori Z, Szelei J, Lacoste MC, et al. A viral phospholipase A2 is required for parvovirus infectivity. Dev Cell 2001;1:291-302.

27. Sonntag F, Bleker S, Leuchs B, et al. Adenoassociated virus type 2 capsids with externalized VP1/VP2 trafficking domains are generated prior to passage through the cytoplasm and are maintained until uncoating occurs in the nucleus. J Virol 2006:80:11040-11054

28. Kurian JJ, Lakshmanan R, Chmely WM, et al. Adeno-associated virus VP1u exhibits protease activity. Viruses 2019;11:399-415.

29. Jin $X$, Liu L, Nass $S$, et al. Direct liquid chromatography/mass spectrometry analysis for complete characterization of recombinant adeno-associated virus capsid proteins. Hum Gene Ther Meth 2017; 28:255-267.

30. Boye SE, Boye SL, Lewin AS, et al. A comprehensive review of retinal gene therapy. Mol Ther 2013:21:509-519.

31. Dalkara D, Sahel JA. Gene therapy for inherited retinal degenerations. C R Biol 2014;337:185-192.

32. Mussolino C, della Corte M, Rossi S, et al. AAVmediated photoreceptor transduction of the pig cone-enriched retina. Gene Ther 2011;18:637-645.

33. Petersen-Jones SM, Occelli LM, Winkler PA, et al. Patients and animal models of CNG $\beta 1$-deficient retinitis pigmentosa support gene augmentation approach. J Clin Invest 2018;128:190-206.

34. Cideciyan AV, Sudharsan R, Dufour VL, et al. Mutation-independent rhodopsin gene therapy by knockdown and replacement with a single AAV vector. PNAS 2019;115:8547-8556.

35. Boye SE, Alexander JJ, Douglas Witherspoon C et al. Highly efficient delivery of adeno-associated viral vector to the primate retina. Hum Gen Ther 2016;27:580-597.

36. Carvalho LS, Xiao R, Wassmer SJ, et al. Synthetic adeno-associated viral vector efficiently targets mouse and nonhuman primate retina in vivo. Hum Gen Ther 2018;29:771-784.

37. Watanabe R, Ueno SS, Hasegawa T, et al. Tropisms of AAV for subretinal delivery to the neonatal mouse retina and its application for in vivo rescue of developmental photoreceptor disorders. PLoS One 2013;8:e54146.

38. Hickey DG, Edwards $T L$, Barnard AR, et al. Tropism of engineered and evolved recombinant AAV serotypes in the rd1 mouse and ex vivo primate retina. Gene Ther 2017;24:787-800.

39. Yin L, Greenberg K, Hunter JJ, et al. Intravitreal injection of AAV2 transduces macaque inner retina. Invest Ophth Vis Sci 2011;52:2775-2783.

40. Wiley LA, Burnight ER, Kaalberg EE, et al Assessment of adeno-associated virus serotype tropism in human retinal explants. Hum Gene Ther 2018;29:424-436.

41. Nass SA, Mattingly MA, Woodcock DA, et al. Universal method for the purification of recombinant AAV vectors of differing serotypes. Mol Ther Meth Clin Dev 2017:9:33-46.
42. Miyazaki J, Takaki S, Araki K, et al. Expression vector system based on the chicken beta-actin promoter directs efficient production of interleukin-5. Gene 1989;79:269-277.

43. Burnham B, Nass $S$, Kong E, et al. Analytical ultracentrifugation as an approach to characterize recombinant adeno-associated viral vectors. Hum Gene Ther Meth 2015;6:228-242.

44. François, A., Bouzelha, M., Lecomte, E., et al. Accurate titration of infectious AAV ParticlesRequires measurement of biologically active vector genomes and suitable controls. Meth Clin Dev 2018:10:223-236.

45. Boye SE, Alexander JJ, Boye SL, et al. The human rhodopsin kinase promoter in an AAV5 vector confers rod- and cone-specific expression in the primate retina. Hum Gene Ther 2012;23:11011115

46. Naidoo J, Stanek LM, Ohno K, et al. Extensive transduction and enhanced spread of a modified AAV2 capsid in the non-human primate CNS. Mol Ther 2018;26:2418-2430.

47. Amado D, Mingozzi F, Hui D, et al. Safety and efficacy of subretinal readministration of a viral vector in large animals to treat congenital blindness. Sci Trans Med 2010;2:21ra16.

48. Kay CN, Ryals RC, Aslanidi GV, et al. Targeting photoreceptors via intravitreal delivery using novel, capsid-mutated AAV vectors. PLoS One 2013; 8:e62097.

49. Llanga T, Bennett W, Woodard K, et al. AAV gene therapy for MPS1-associated corneal blindness. Sci Rep 2016;6:22131-22141

50. Sharma A, Tovey JC, Ghosh A, et al. AAV serotype influences gene transfer in corneal stroma in vivo. Exp Eye Res 2010;91:440-448.

51. Rubentein PA, Wen KK. NATure of actin aminoterminal acetylation. PNAS 2018;115:43144316

52. Behnia R, Panic B, Whyte JR, et al. Targeting of the Arf-like GTPase Arl3p to the Golgi requires $\mathrm{N}$-terminal acetylation and the membrane protein Sys1p. S Nat Cell Biol 2004;6:403-413.

53. Lewis JJ, Singh RNP, Bones OF, et al. Altered proteolytic cleavage of human growth hormone as a result of deamidation. J Biol Chem 1981;256: $11645-11650$

54. Giles AR, Sims JJ, Turner KB, et al. Deamidation of amino acids on the surface of adeno-associated virus capsids leads to charge heterogeneity and altered vector function. Mol Ther 2018;26:28482862

55. Hordeaux J, Wang Q, Katz N, et al. The neurotropic properties of AAV-PHP.B are limited to C57BL/6J Mice. Mol Ther 2018;26: $664-668$

Received for publication March 29, 2020; accepted after revision June 3, 2020.

Published online: June 13, 2020 